Article

\title{
Development of a Semi-Analytical Algorithm for the Retrieval of Suspended Particulate Matter from Remote Sensing over Clear to Very Turbid Waters
}

\author{
Bing Han 1,2,3 , Hubert Loisel 2,4,5,*, Vincent Vantrepotte ${ }^{2,6}$, Xavier Mériaux ${ }^{2}$, Philippe Bryère ${ }^{7}$, \\ Sylvain Ouillon ${ }^{4,8}$, David Dessailly ${ }^{2}$, Qianguo Xing ${ }^{9}$ and Jianhua Zhu ${ }^{1}$ \\ 1 National Ocean Technology Center (NOTC), 219 Jieyuanxi Rd., Tianjin 300112, China; \\ binghanrs@126.com (B.H.); besmile@263.net (J.Z.) \\ 2 Université du Littoral Côte d'Opale (ULCO), Laboratoire d'Océanologie et de Géosciences (LOG), \\ 62930 Wimereux, France; vincent.vantrepotte@univ-littoral.fr (V.V.); xavier.meriaux@univ-littoral.fr (X.M.); \\ david.dessailly@univ-littoral.fr (D.D.) \\ 3 School of Marine Sciences, Nanjing University of Information Science and Technology (NUIST), \\ 219 Jingning 6 Rd., Nanjing210044, China \\ 4 Institut de Recherche Pour le Développement (IRD), Université de Toulouse, UPS (OMP), UMR 5566 LEGOS, \\ 14 av. Edouard Belin, 31400 Toulouse, France; sylvain.ouillon@legos.obs-mip.fr \\ 5 Space Technology Institute (STI), Vietnam Academy of Science \& Technology (VAST), 18 Hoang Quoc Viet, \\ CauGiay, Hanoi, Vietnam \\ 6 CNRS Guyane, USR3456, 97334 Cayenne Cedex, France \\ 7 ACRI-HE, 8 Quai de la douane, 29200 Brest, France; Philippe.Bryere@acri-he.fr \\ 8 Department Water-Environment-Oceanography, University of Science and Technology of Hanoi (USTH), \\ 18 Hoang Quoc Viet, CauGiay, Hanoi, Vietnam \\ 9 Yantai Institute of Coastal Zone Research (YIC), Chinese Academy of Sciences, 17 Chunhui Rd, \\ Yantai 264003, China; qgxing@yic.ac.cn \\ * Correspondence: hubert.loisel@univ-littoral.fr; Tel.: +33-321-99-6420
}

Academic Editors: Deepak R. Mishra, Richard W. Gould Jr., Xiaofeng Li and Prasad S. Thenkabail Received: 26 December 2015; Accepted: 29 February 2016; Published: 5 March 2016

\begin{abstract}
Remote sensing of suspended particulate matter, SPM, from space has long been used to assess its spatio-temporal variability in various coastal areas. The associated algorithms were generally site specific or developed over a relatively narrow range of concentration, which make them inappropriate for global applications (or at least over broad SPM range). In the frame of the GlobCoast project, a large in situ data set of SPM and remote sensing reflectance, $R_{\mathrm{rs}}(\lambda)$, has been built gathering together measurements from various coastal areas around Europe, French Guiana, North Canada, Vietnam, and China. This data set covers various contrasting coastal environments diversely affected by different biogeochemical and physical processes such as sediment resuspension, phytoplankton bloom events, and rivers discharges (Amazon, Mekong, Yellow river, MacKenzie, etc.). The SPM concentration spans about four orders of magnitude, from 0.15 to $2626 \mathrm{~g} \cdot \mathrm{m}^{-3}$. Different empirical and semi-analytical approaches developed to assess SPM from $R_{\mathrm{rs}}(\lambda)$ were tested over this in situ data set. As none of them provides satisfactory results over the whole SPM range, a generic semi-analytical approach has been developed. This algorithm is based on two standard semi-analytical equations calibrated for low-to-medium and highly turbid waters, respectively. A mixing law has also been developed for intermediate environments. Sources of uncertainties in SPM retrieval such as the bio-optical variability, atmospheric correction errors, and spectral bandwidth have been evaluated. The coefficients involved in these different algorithms have been calculated for ocean color (SeaWiFS, MODIS-A/T, MERIS/OLCI, VIIRS) and high spatial resolution (LandSat8-OLI, and Sentinel2-MSI) sensors. The performance of the proposed algorithm varies only slightly from one sensor to another demonstrating the great potential applicability of the proposed approach over global and contrasting coastal waters.
\end{abstract}


Keywords: suspended particulate matter; coastal waters; ocean color; specific backscattering coefficient; empirical algorithm; semi-analytic algorithm

\section{Introduction}

Monitoring suspended particulate matter (SPM, see Table 1 for symbols and acronyms) spatio-temporal distribution in coastal waters is of particular importance for a variety of applications dedicated to coastal management which often implicitly contain an economic interest. This for instance includes studies aiming to evaluate the impact of human activities (e.g., dam, offshore wind turbine farms, sand extraction) on the sediment transport, downstream sedimentation, and coastal geo-morphological processes (accretion, erosion) occurring in a defined coastal region [1,2]. Forecasting SPM dynamics in response to natural (river discharges, tidal current, waves, etc.) or anthropogenic forcing is at the same time of high interest for optimizing human efforts related to unbalanced sediments stocks as, for instance, for better anticipating dredging activities in areas affected by coastal environmental changes. The latter applications are often based on the development of sediment transport models for which reliable information on SPM variability represents a crucial input for the adjustment of some key parameters [3-5]. Besides, as a major controlling factor of light and nutrient availability in coastal and estuarine waters [6], knowledge on SPM dynamics is also important for the understanding of ecosystems structure and functioning. Last but not least, information on particulate matter in water masses is also crucial for better constraining water quality or pollution $[7,8]$ or studying major biogeochemical cycles $[9,10]$.

Table 1. List of symbols and acronyms.

\begin{tabular}{clc}
\hline Symbol/Acronym & \multicolumn{1}{c}{ Description } & Unit \\
\hline $\mathrm{SPM}$ & Suspended particulate matter concentration & $\mathrm{g} \cdot \mathrm{m}^{-3}$ \\
$\mathrm{R}_{\mathrm{rs}}$ & Remote sensing reflectance & $\mathrm{sr}^{-1}$ \\
$\mathrm{R}$ & Irradiance reflectance & dimensionless \\
$\mathrm{Q}$ & Bidirectionality factor & $\mathrm{sr}$ \\
$\rho_{\mathrm{w}}$ & Water leaving reflectance & dimensionless \\
$\lambda$ & Wavelength & $\mathrm{nm}$ \\
$\mathrm{a}$ & Total water absorption & $\mathrm{m}^{-1}$ \\
$\mathrm{a}_{\mathrm{w}}$ & Pure water absorption & $\mathrm{m}^{-1}$ \\
$\mathrm{a}_{\mathrm{p}}$ & Particulate absorption coefficient & $\mathrm{m}^{-1}$ \\
$\mathrm{~b}_{\mathrm{bp}}$ & Particulate backscattering coefficient & $\mathrm{m}^{-1}$ \\
$\mathrm{~b}_{\mathrm{bp}}^{*}$ & Mass-specific particulate backscattering coefficient & $\mathrm{m}^{2} \cdot \mathrm{g}^{-1}$ \\
$\mathrm{nLvSM}$ & non-living suspended particulate matter & $\mathrm{g}^{-3} \mathrm{~m}^{-3}$ \\
$\mathrm{Chl}$ & Chlorophyll-a concentration & $\mathrm{mg}^{-3} \mathrm{~m}^{-3}$ \\
$\mathrm{IOP}$ & Inherent optical property & - \\
$\mathrm{SRF}$ & Spectral Response Function & - \\
$\mathrm{DS}-\mathrm{T}$ & Whole data set present in this study & - \\
DS-D/V & Sub-dataset of DS-T for model development/validation & - \\
DS-IOP & Sub-dataset of DS-T where IOP is available & - \\
EA-MB & Multi-Bands Empirical Algorithms & - \\
EA-BR & Simple Band-Ratio Empirical Algorithms & - \\
SAA & semi-analytical algorithm & - \\
\hline
\end{tabular}

This overall environmental and economic importance of mapping SPM distribution in coastal waters has motivated the development of numerous inversion algorithms in recent years for estimating quantitatively SPM concentration from the remote sensing reflectance, $R_{\mathrm{rs}}(\lambda)$ (in sr ${ }^{-1}$ ). Ocean color remote sensing indeed offers the possibility to map SPM and monitor its dynamics over different temporal and spatial scales, in particular, taking advantage of the complementary 
information now provided by the possible combination of medium (e.g., MERIS, MODIS, SeaWiFS, VIIRS) and high spatial resolution sensors (e.g., LandSat, SPOT, Sentinel-2) measurements. The algorithms needed to estimate SPM load from the marine reflectance can be divided into three major categories including empirical, semi-analytical or analytical models. First, a variety of empirical formulations diversely based on different spectral band combinations have been developed within different coastal sites. Single band formulations based on various spectral inputs have been, for instance, widely documented [4,11-18]. The major issue related to these straightforward models is related to their frequent saturation in the most turbid waters due to the asymptotical shape of the link between the marine reflectance signal at a defined wavelength and SPM concentration. To overcome this issue, as well as to reduce the impact of atmospheric correction errors, several band ratios [4,19-22] or multiband [1,16,22-24] models have been proposed. Further, various semi-analytical models based on a theoretical model, often simplified, have been also proposed for estimating SPM loads from the marine reflectance via information on particulate matter Inherent Optical Properties (IOPs) [25-31]. Finally, SPM concentration can be derived from theoretical radiative transfer models generating the top-of-atmosphere spectra with varying water (including SPM loads) and atmospheric conditions [32,33]. Each of the latter models, however, contains its specific limitation. Those using empirical relationships are strongly dependent on the SPM and $R_{\mathrm{rs}}$ ranges over which they have been developed. This often leads in practice to site-specific models whose coefficients need to be locally adapted for a defined coastal domain. Conversely, the performance of the models partly or fully based on a theoretical link between SPM and IOPs is strongly conditioned by the representativeness of the hypothesis chosen for describing particulate backscattering or absorption properties which are expected to vary widely according to the nature of the particulate matter pool [34-37].

Facing this multiplicity of possible models, the choice of the one most appropriated for a defined region is therefore often challenging. Further, the expected specific limitations of the existing models also prevent the development of large scale studies dedicated to particulate matter dynamics in coastal waters. Finally, documented models are usually developed for a defined sensor and thus need to be adapted in order to take into account sensor specific spectral characteristics such as spectral resolution, signal to noise ratio or sensors spectral response.

This study was built in this context and aimed at improving the retrieval of SPM concentration for global scale applications in coastal waters (i.e., over large SPM concentration range). For this purpose, an extensive in situ data set $(N=631)$ of coincident bio-optical ( $R_{\mathrm{rs}}$ and IOPs) and SPM measurements was built gathering measurements collected within five contrasted coastal sites (European coastal waters, French Guyana, Eastern Vietnam sea, China Yellow sea, and North Canada). Based on this data set covering more than four orders of magnitude in SPM concentration, the first objective is to precisely assess the potential limitations of existing historical empirical and semi-analytical models. Considering that an exhaustive comparison of the available SPM inversion models is not achievable in practice, four models initially developed based on contrasting hypotheses are considered: two empirical formulations [20,24] and two semi-analytical models [26,29]. In a second step, generalized formulations for retrieving SPM concentrations over this wide range of turbidity and for a variety of ocean color (i.e., SeaWiFS, MODIS-A/T, MERIS/OLCI, VIIRS) and high resolution (LandSat 8-OLI, and Sentinel 2-MSI) sensors are proposed. Finally, a sensitivity analysis aiming to identify the source of error in SPM retrieval is specifically investigated from the present extended in situ data set for the different models proposed. The impact of the natural variability in particulate matter IOPs, the uncertainties in the satellite reflectance signal, and also the impact of sensors specific spectral response are specifically investigated from this extended in situ data set. These different steps are summarized in Figure 1. 


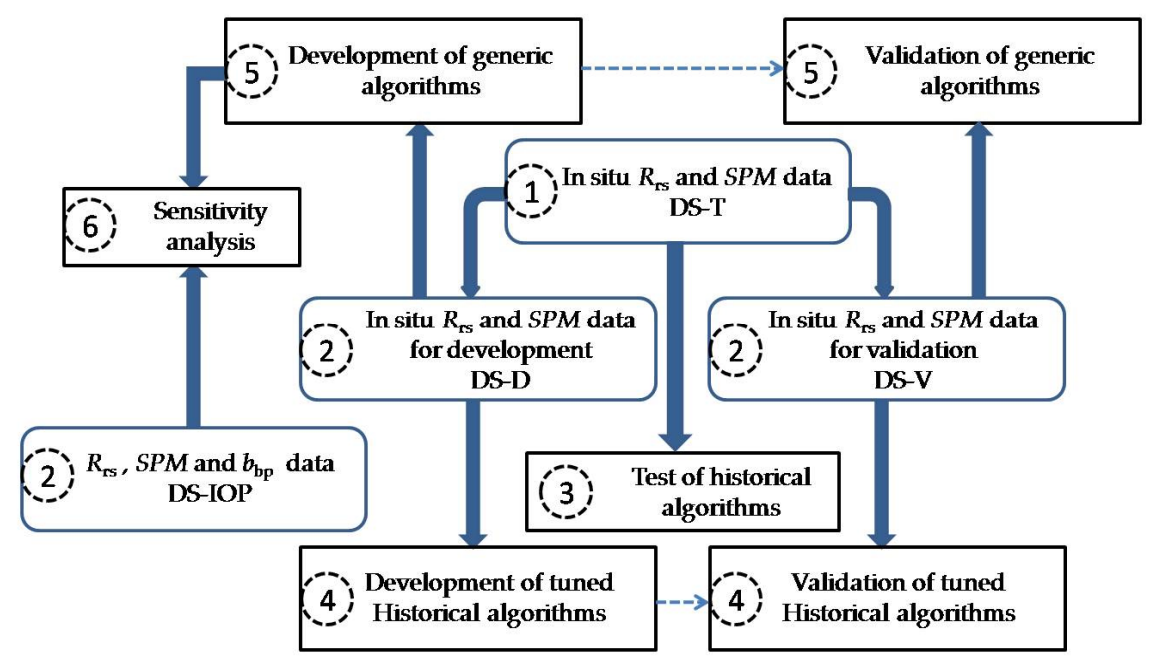

Figure 1. Flowchart of the present work. Number in each box indicates the different steps of the study, while rectangles in black stand for activities and round-corner rectangle in blue for data set.

\section{Materials and Methods}

\subsection{Data}

The present data set gathers in situ measurements of $S P M$ and $R_{\mathrm{rs}}(\lambda)$ collected in various coastal regions (European coastal waters, French Guiana, Eastern Vietnam sea, China Yellow sea, and North Canada) within the framework of different oceanographic field surveys (Figure 2).

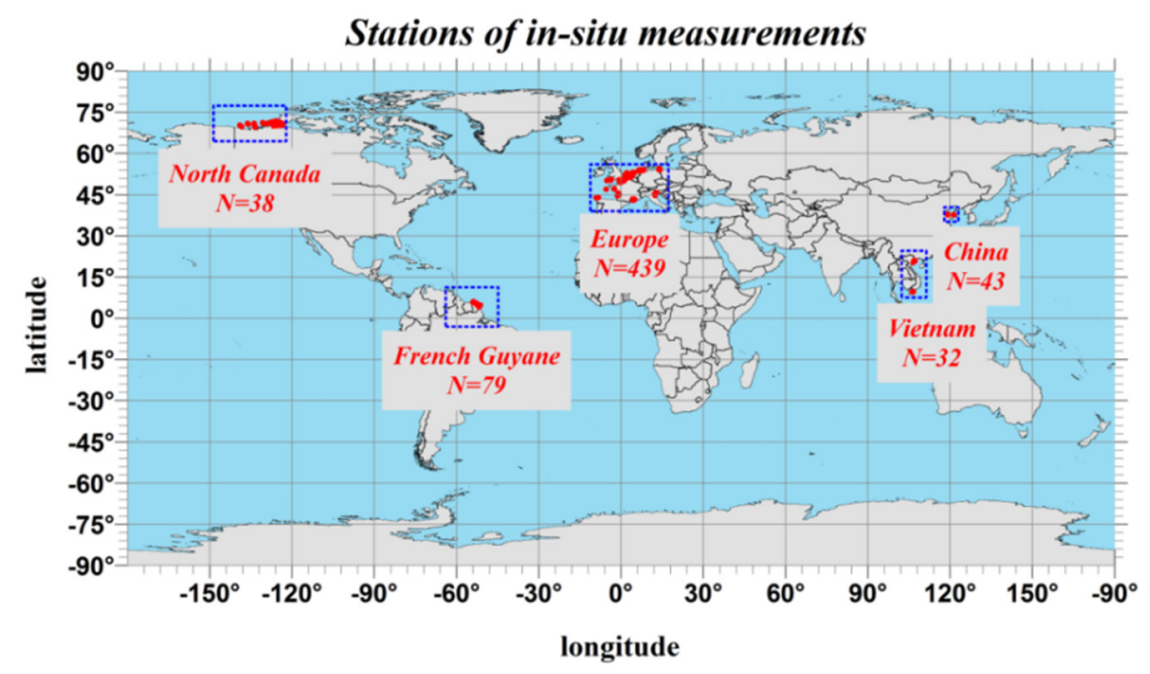

Figure 2. Location of the in situ data points used for the development and validation of the different $S P M$ algorithms. $\mathrm{N}$ represents the number of in situ $\left(R_{\mathrm{rs}}(\lambda), S P M\right)$ data points for each sampled region.

This data set covers many different physical and biological coastal environments which drastically differ in terms of sources and sinks of SPM (i.e., river run-off, sediment resuspension, phytoplankton blooms, etc.), and covers the natural variability both in terms of SPM range and chemical nature (organic vs. mineral).Six hundred and thirty-one (from 707) pairs of $\left(R_{\mathrm{rs}}(\lambda), S P M\right)$ data points were kept after data quality controls based on the application of several criteria such as unusual $R_{\mathrm{rs}}$ spectra shapes, negative values in the near infra-red, and strong deviation from the scatter pattern around the $R_{\mathrm{rs}}$ vs.SPM relationship in the red. This whole data set will be referenced as DS-T in the following parts of this work and gathers $S P M$ varying between 0.15 and $2626 \mathrm{~g} \cdot \mathrm{m}^{-3}$. Among the $R_{\mathrm{rs}}(\lambda)$ spectra 
in DS-T, 311 (about 49\%) were obtained from hyperspectral measurements covering the (350 nm, $900 \mathrm{~nm}$ ) spectral range, and the others from multispectral measurements at the usual ocean color wavebands. The field measurements' protocol and the data processing of $R_{\mathrm{rs}}(\lambda)$ and SPM can be obtained in Babin et al. (2003) [38], Doron et al. (2006) [39], Lubac and Loisel (2007) [40], Bélanger et al. (2008) [41], and Nechad et al. (2010) [26]. While all SPM measurements have been performed using the same protocol as the one presented in Babin et al. [38] based on GF/F filters, $R_{\mathrm{rs}}(\lambda)$ spectra were obtained from in-water or above-water radiometric measurements. The calculation of $R_{\mathrm{rs}}(\lambda)$ from these measurements has been performed using the standard protocols for above-water [42] and in-water [43] radiometric measurements. $R_{\mathrm{rs}}(\lambda)$ spectra for some sampling stations around Europe were obtained from irradiance reflectance, $R(\lambda)$ using the approach of Doron et al. [39] which includes the Q-factor as calculated by Loisel and Morel [44] for coastal waters.The DS-T data set was then randomly separated into two parts, i.e., a development dataset (DS-D) and a validation dataset (DS-V), which represent $70 \%$ and $30 \%$ of the data in DS-T, respectively. DS-D and DS-V show consistent statistics which proves a reasonable division. They both cover more than about four orders of magnitude in terms of SPM, allowing the natural variability of coastal environment to be satisfactorily represented (Figure 3a,b).
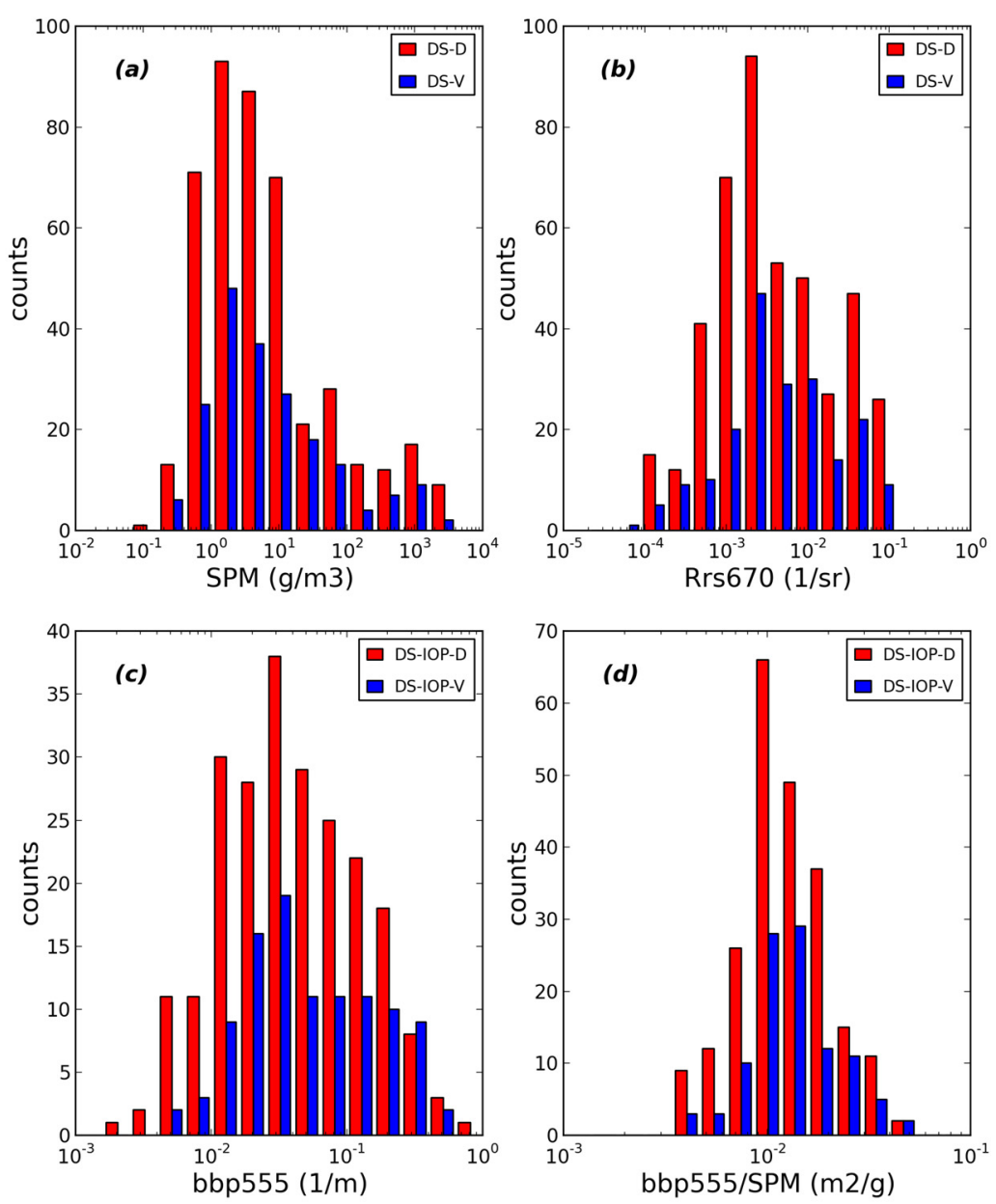

Figure 3. Frequency distribution of (a) $S P M$; (b) $R_{\mathrm{rs}}(670)$; (c) $b_{\mathrm{bp}}(555)$; and (d) $b_{\mathrm{bp}}(555) / S P M$ in terms of logarithmically equal interval. The data used for the development and validation are represented in red and blue, respectively.

In open ocean waters, the variability of the chlorophyll specific particulate backscattering coefficient is known to have a great impact on the retrieval accuracy of the Chlorophyll concentration, $\mathrm{Chl}$, from ocean color remote sensing algorithms [45]. Similarly, the impact of the mass particulate 
specific backscattering coefficient, $b_{\mathrm{bp}} / \mathrm{SPM}$ (noted as $b_{b p}^{*}$ ), in $\mathrm{m}^{2} \cdot \mathrm{g}^{-1}$, on the accuracy of SPM retrieval will be specifically assessed. For that purpose, a third sub-dataset (denoted as DS-IOP) gathering $S P M, R_{\mathrm{rs}}(\lambda), b_{\mathrm{bp}}$, and $b_{b p}^{*}$ data points has been generated (Table 2 ). The processing of the $b_{\mathrm{bp}}$ data is described in Lubac and Loisel (2007) [40]. For the very turbid waters, defined as SPM concentration higher than $50 \mathrm{~g} \cdot \mathrm{m}^{-3}$, the standard protocols developed by the manufacturer (Wetlabs) to assess $b_{\mathrm{bp}}$ from scattering measurements performed by the different instruments (BB-9 and ECO-VSF) can lead to an over-estimation of $b_{\mathrm{bp}}$. For this reason, and before the development of a proper methodology which should encompass scattering correction along the pathlength of the instrument, data points that deviate too much from the $b_{\mathrm{bp}}$ vs. SPM relationship developed in Neukermans et al. [37] have been disregarded. Six pairs of $\left(b_{\mathrm{bp}}, S P M\right)$ data points with SPM higher than $50 \mathrm{~g} \cdot \mathrm{m}^{-3}$ are present in DS-IOP. DS-IOP is also randomly divided into two parts (i.e., DS-IOP-D and DS-IOP-V) with 202 and 106 data points, respectively. These two sub-data sets cover the same range for each parameter (example for $b_{\mathrm{bp}}$ and $b_{\mathrm{bp}} / \mathrm{SPM}$ in Figure $\left.3 \mathrm{c}, \mathrm{d}\right)$, and are representative of coastal waters with the exception of highly turbid waters.

Table 2. Mean, standard deviation, minimum, and maximum values of $S P M, R_{\mathrm{rs}}(670), b_{\mathrm{bp}}(488)$ and $b_{\mathrm{bp}}^{*}(488)$ for each specific regions. $\mathrm{N}$ represents the number of measurements in each sub-region.

\begin{tabular}{|c|c|c|c|c|}
\hline & $S P M$ & $R_{\mathrm{rs}}(670)$ & $b_{\mathrm{bp}}(488)^{1}$ & $b_{\mathrm{bp}}^{*}(488)^{\mathbf{1}}$ \\
\hline & $\left(g \cdot m^{-3}\right)$ & $\left(\mathbf{s r}^{-1}\right)$ & $\left(m^{-1}\right)$ & $\left(m^{2} \cdot g^{-1}\right)$ \\
\hline Europe & $\begin{array}{c}54.37 \pm 248.75 \\
(0.154,2627) \\
N=439\end{array}$ & $\begin{array}{c}0.0081 \pm 0.0141 \\
(0.0001,0.0663) \\
N=439\end{array}$ & $\begin{array}{c}0.0780 \pm 0.1230 \\
(0.0034,0.84) \\
N=248\end{array}$ & $\begin{array}{c}0.0165 \pm 0.0098 \\
(0.0038,0.051) \\
N=248\end{array}$ \\
\hline French Guiana & $\begin{array}{c}64.68 \pm 115.096 \\
(1.520,604.5) \\
N=79\end{array}$ & $\begin{array}{c}0.0176 \pm 0.0133 \\
(0.0012,0.045) \\
N=79\end{array}$ & $\begin{array}{c}0.2024 \pm 0.166 \\
(0.0308,0.736) \\
N=50\end{array}$ & $\begin{array}{c}0.0141 \pm 0.004 \\
(0.0059,0.0235) \\
N=50\end{array}$ \\
\hline Vietnam & $\begin{array}{c}12.35 \pm 13.75 \\
(2.020,72.8) \\
N=32\end{array}$ & $\begin{array}{c}0.0064 \pm 0.006 \\
(0.0016,0.031) \\
N=32\end{array}$ & $\begin{array}{c}0.1255 \pm 0.144 \\
(0.0232,0.81) \\
N=30\end{array}$ & $\begin{array}{c}0.0111 \pm 0.003 \\
(0.0064,0.02) \\
N=30\end{array}$ \\
\hline North Canada & $\begin{array}{c}1.90 \pm 3.77 \\
(0.207,17.82) \\
N=38\end{array}$ & $\begin{array}{c}0.0015 \pm 0.003 \\
(0.0001,0.016) \\
N=38\end{array}$ & - & - \\
\hline China & $\begin{array}{c}748.754 \pm 7714.3 \\
(6.300,2435.4) \\
N=43\end{array}$ & $\begin{array}{c}0.0574 \pm 0.026 \\
(0.0023,0.095) \\
N=43\end{array}$ & - & - \\
\hline DS-T & $\begin{array}{c}97.691 \pm 331.6 \\
(0.154,2627) \\
N=631\end{array}$ & $\begin{array}{c}0.0122 \pm 0.02 \\
(0.0001,0.095) \\
N=631\end{array}$ & $\begin{array}{c}0.1013 \pm 0.14 \\
(0.0034,0.84) \\
N=328\end{array}$ & $\begin{array}{c}0.0156 \pm 0.009 \\
(0.0038,0.051) \\
N=328\end{array}$ \\
\hline
\end{tabular}

\subsection{Selection of Algorithm Families}

Empirical and semi-analytical algorithms were developed from DS-D while their performance was evaluated on DS-V. Based on previous studies, two empirical algorithms and two semi-analytical models have been selected. While many models have been developed in the literature to assess SPM from $R_{\mathrm{rs}}$, the choice of these four models was motivated by the fact that they are representative of the different approaches found in the literature: purely empirical approaches using different spectral bands; or semi-analytical algorithms based on the use of one or several bands combined with assumption on IOPs. The Multi-Bands Empirical Algorithms, EA-MB, follow the formulation originally developed by Tassan et al. (1994) [22], and recently revisited by Siswanto et al. (2011) [24] and Loisel et al. (2014) [1]. Simple Band-Ratio Empirical Algorithms, EA-BR, were already developed [4,19,20,46] and applied 
to high spatial resolution sensors, such as SPOT [4] or medium resolution sensors (e.g., MODIS, Doxaran et al., 2015 [47]). Note: here $E A$ stands for empirical, $M B$ for multiband, and $B R$ for band ratio. Two different semi-analytical approaches are also tested. The first one is based on a relationship between non-living suspended particulate matter, $n L v S P M, S P M$, and the Chlorophyll-a concentration, Chl (Gohin et al., 2005 [29]). nLvSPM is estimated from the normalized water leaving radiance at $555 \mathrm{~nm}$ or $670 \mathrm{~nm}$ (depending on the level of turbidity), using the two step algorithm presented in Gohin et al. (2005) [29]. The second semi-analytical algorithm, SAA, is based on the well-known $R_{\mathrm{rs}}$ vs. IOPs relationship in the red part of the spectrum (Nechad et al., 2010 [26]). In practice, the following original algorithms from the latter four distinct families have been tested within the framework of this study:

(1) EA-MB (Siswanto et al., 2011 [24])

$$
\log _{10}(\mathrm{SPM})=\mathrm{c}_{0}+\mathrm{c}_{1} \cdot \mathrm{X}_{1} \cdot+\mathrm{c}_{2} \cdot \mathrm{X}_{2}
$$

where $\mathrm{X}_{1}=R_{\mathrm{rs}}\left(\lambda_{1}\right)+R_{\mathrm{rs}}\left(\lambda_{2}\right)$ is the sensitive term to $S P M, \mathrm{X}_{2}=R_{\mathrm{rs}}\left(\lambda_{3}\right) / R_{\mathrm{rs}}\left(\lambda_{1}\right)$ serves as a compensating term, and $\lambda_{1}, \lambda_{2}$ and $\lambda_{3}$ are in the green, red and blue regions of the visible spectrum, respectively. For the SeaWiFS sensor, $\lambda_{1}, \lambda_{2}$ and $\lambda_{3}$ are $555 \mathrm{~nm}, 670 \mathrm{~nm}$ and $490 \mathrm{~nm}$, respectively.

(2) EA-BR (Doxaran et al., 2003 [20])

$$
S P M=\mathrm{a}_{0} \cdot \exp \left(\mathrm{a}_{1} \cdot \mathrm{X}\right)
$$

where $\mathrm{X}=R_{\mathrm{rs}}\left(\lambda_{1}\right) / R_{\mathrm{rs}}\left(\lambda_{2}\right)$, with $\lambda_{1}$ and $\lambda_{2}$ are in the near-infrared and green part of the spectrum (e.g., $865 \mathrm{~nm}$ and $555 \mathrm{~nm}$ for SeaWiFS), respectively.

(3) SAA(Nechad et al., 2010 [26])

$$
S P M=\mathrm{A}^{\rho} \cdot \rho_{\mathrm{w}}\left(\lambda_{0}\right) /\left(1-\rho_{\mathrm{w}}(670) / \mathrm{C}^{\rho}\right)+\mathrm{B}^{\rho}
$$

where $\rho_{\mathrm{w}}\left(\lambda_{0}\right)=\pi \cdot R_{\mathrm{rs}}\left(\lambda_{0}\right)$ is the water leaving reflectance, $A^{\rho}\left(\mathrm{in} \mathrm{g} \cdot \mathrm{m}^{-3}\right.$ ) and $C^{\rho}$ (dimensionless) are related to IOPs, while $B^{\rho}$ accounts for uncertainties in the measurements. $A^{\rho}$ is the ratio of non-algal particulate absorption to the specific particulate backscattering coefficient, $b_{\mathrm{bp}} / S P M$, and $C^{\rho}$ is the ratio of $b_{\mathrm{bp}} / S P M$ to the specific particulate absorption, $a_{\mathrm{p}} / S P M$. This analytical equation can be directly obtained from the proportionality of $R_{\mathrm{rs}}$ with the $b_{\mathrm{b}} /\left(a+b_{\mathrm{b}}\right)$ ratio, assuming that the total absorption coefficient, $a$, is dominated by pure sea water absorption, $a_{\mathrm{w}}$, in the red part of the spectrum.

(4) Gohin (Gohin et al., 2005 [29]; Gohin, 2011 [48])

$$
S P M=n L v S P M+234 \cdot C h l^{0.57}
$$

where Chl is estimated from OC5 (Gohin et al., 2010 [49]) and nLvSPM is calculated from a two steps algorithm from the reflectance in the green or in the red, and assumptions about specific IOPs (their Table 3 in Gohin et al., 2005 [29]). The reflectance in the red is used for waters presenting SPM concentration higher than $4 \mathrm{~g} \cdot \mathrm{m}^{-3}$ (Gohin, 2011 [48]). Specific look-up table (to estimate Chlorophyll-a concentration) and the coefficients for the equations are needed for the computation of SPM.

After being tested on the DS-T data set, these selected algorithms were modified (except the Gohin et al. (2005) [29] algorithm which cannot be easily modified) based on the DS-D data set which covers a large range of SPM variability. The coefficients in Equations (1)-(3) were determined based on the condition that the cost function is minimized, this function being defined as the sum of squared difference between measured and estimated values (least square technique). However, for SAA, a non-linear regression, was applied and the cost function was calculated based on log-transformed values, considering that bio-optical parameters of oceanic waters are log-normally distributed [50]. 
Table 3. Values of the parameters involved in the different models calibrated on the development sub-data set, DS-D.

\begin{tabular}{|c|c|c|c|c|}
\hline Model Index & Wavebands Involved & Re-Calibrated Model Parameters & $\mathbf{N}$ & $R^{2}$ \\
\hline$E A-M B$ & \multirow{3}{*}{$\left(\lambda_{1}, \lambda_{2}, \lambda_{3}\right)=(555,670,490 \mathrm{~nm})$} & $\left(c_{0}, c_{1}, c_{2}\right)=(0.440,24.083,-0.397)$ & 435 & 0.90 \\
\hline $\begin{array}{c}E A-M B-L \\
\left(S P M<100 \mathrm{~g} \cdot \mathrm{m}^{-3}\right)\end{array}$ & & $\left(c_{0}, c_{1}, c_{2}\right)=(0.451,22.674,-0.399)$ & 384 & 0.89 \\
\hline $\begin{array}{c}E A-M B-H \\
\left(S P M \geqslant 100 \mathrm{~g} \cdot \mathrm{m}^{-3}\right)\end{array}$ & & $\left(c_{0}, c_{1}, c_{2}\right)=(1.047,13.139,-0.590)$ & 51 & 0.78 \\
\hline$S A A$ & \multirow{3}{*}{$\lambda_{0}=670 \mathrm{~nm}$} & $\left(A^{\rho}, C^{\rho}\right)=(428.277,0.3051)$ & 435 & - \\
\hline $\begin{array}{c}S A A-L \\
\left(S P M<100 \mathrm{~g} \cdot \mathrm{m}^{-3}\right)\end{array}$ & & $\left(A^{\rho}, C^{\rho}\right)=(391.082,0.5000)$ & 384 & - \\
\hline $\begin{array}{c}S A A-H \\
\left(S P M \geqslant 100 \mathrm{~g} \cdot \mathrm{m}^{-3}\right)\end{array}$ & & $\left(\mathrm{A}^{\rho}, \mathrm{C}^{\rho}\right)=(1444.853,0.3539)$ & 51 & - \\
\hline$E A-B R$ & \multirow{3}{*}{$\left(\lambda_{1}, \lambda_{2}\right)=(865 \mathrm{~nm}, 555 \mathrm{~nm})$} & $\left(a_{0}, a_{1}\right)=(194.391,0.909)$ & 253 & 0.65 \\
\hline $\begin{array}{c}E A-B R-L \\
\left(S P M<100 \mathrm{~g} \cdot \mathrm{m}^{-3}\right)\end{array}$ & & $\left(a_{0}, a_{1}\right)=(30.878,0.501)$ & 202 & 0.34 \\
\hline $\begin{array}{c}E A-B R-H \\
\left(S P M \geqslant 100 \mathrm{~g} \cdot \mathrm{m}^{-3}\right)\end{array}$ & & $\left(a_{0}, a_{1}\right)=(760.181,1.307)$ & 51 & 0.70 \\
\hline
\end{tabular}

\subsection{Statistical Indicators}

Four statistical indicators were used to characterize the performance of the different models. The average relative percentage error (Bias), mean relative absolute difference (MRAD), and average ratio of estimated to measured values (ratio) are calculated based on original values. Since the bio-optical variability at a variety of spatial and temporal scales is generally considered to be log-normally distributed [50], root mean square error (RMSE_log) was calculated in log space. Besides, as SPM spans over four orders of magnitude, the RMSE in linear space is highly impacted by extremely large values, which totally bias the meaning of RMSE. These indicators are calculated as follows:

$$
\begin{gathered}
\text { Bias }=100 \% \cdot \frac{1}{N} \sum_{i=1}^{N} \frac{y_{i}-y_{i}^{\prime}}{y_{i}} \\
\text { MRAD }=100 \% \cdot \frac{1}{N} \sum_{i=1}^{N} \frac{\left|y_{i}-y_{i}^{\prime}\right|}{y_{i}} \\
\text { ratio }=\frac{1}{N} \sum_{i=1}^{N} \frac{y_{i}^{\prime}}{y_{i}} \\
\text { RMSE_log }=\sqrt{\frac{1}{N} \sum_{i=1}^{N}\left[\log _{10}\left(y_{i}^{\prime}\right)-\log _{10}\left(y_{i}\right)\right]^{2}}
\end{gathered}
$$

where $y$ and $y^{\prime}$ are measured and estimated values, respectively. $N$ is total number of records.

\section{Results and Discussion}

\subsection{General Trends between $R_{r s}$ and $S P M$}

The correlation between $R_{\mathrm{rs}}$ and SPM was analyzed in logarithmic scale for the wavelengths available on current ocean color sensors prior to the development and validation of the SPM algorithms (Figure 4). As already mentioned in previous studies [12,26], the correlation coefficient between $R_{\mathrm{rs}}$ and SPM increases from the blue to the red spectral domain. For instance, the determination coefficient, $R^{2}$, increases from 0.563 at $490 \mathrm{~nm}$ (Figure 4a) to 0.775 at $765 \mathrm{~nm}$ (Figure 4e). In the same way, the scatter observed around the $R_{\mathrm{rs}} v s . S P M$ relationship continuously decreases from the blue to the red 
part of the spectrum. These two observations can be related to the increase with the wavelength of the relative contribution of pure water absorption to the total absorption coefficient, as well as to the increasing influence of the particulate backscattering coefficient, $b_{\mathrm{bp}}$, on the $R_{\mathrm{rs}}$ variability. While the $R_{\mathrm{rs}}$ values measured in the visible part of the spectrum tend to saturate from SPM values of about $50 \mathrm{~g} \cdot \mathrm{m}^{-3}$ (Figure $4 \mathrm{c}, \mathrm{d}$ ), the linearity between $\log \left(R_{\mathrm{rs}}\right)$ and $\log (S P M)$ is recovered in the near-infrared part of the spectrum (Figure 4e,f). This is consistent with previous studies $[4,19,20,51]$ and can be explained by the fact that pure water absorption, $a_{\mathrm{w}}$, increases by almost a factor of 10 from the red to the NIR part of the spectrum. For highly turbid waters, as $R_{\mathrm{rs}}$ can be approximated by the $b_{\mathrm{b}} /\left(a+b_{\mathrm{b}}\right)$ ratio, $R_{\mathrm{rs}}$ is only driven by $b_{\mathrm{bp}}$ in the NIR, whereas this ratio (and thus $R_{\mathrm{rs}}$ ) does not sharply increase in the red, as $b_{\mathrm{b}}$ and $a$ are globally at the same order of magnitude. Note however that, while the slope in the $\log \left(R_{\mathrm{rs}}(670)\right)$ vs. $\log (S P M)$ relationship changes from turbid to highly turbid waters (Figure $\left.4 \mathrm{~d}\right)$, $R_{\mathrm{rs}}(670)$ is still sensitive to $S P M$ variability in such very turbid environments. This feature is important, considering that the accuracy in the $R_{\mathrm{rs}}$ estimate in the NIR has still not been fully evaluated, creating therefore potential doubt in the use of such NIR channel for evaluating SPM concentration. In the same way, the correlation between SPM and four different $R_{\mathrm{rs}}$ band ratios (all with respect to the green band, i.e., $555 \mathrm{~nm}$ for SeaWiFS) has also been examined (Figure 5). Among the four band ratios investigated here, the red-to-green band ratio, $R_{\mathrm{rs}}(670) / R_{\mathrm{rs}}(555)$, is the one which exhibits the best correlation with SPM when the full SPM range is considered. Similar to the case of a single $R_{\mathrm{rs}}$ band, near-infrared-to-green band ratios such as $R_{\mathrm{rs}}(765) / R_{\mathrm{rs}}(555)$ and $R_{\mathrm{rs}}(865) / R_{\mathrm{rs}}(555)$ are only well related with SPM for highly and extremely turbid waters.
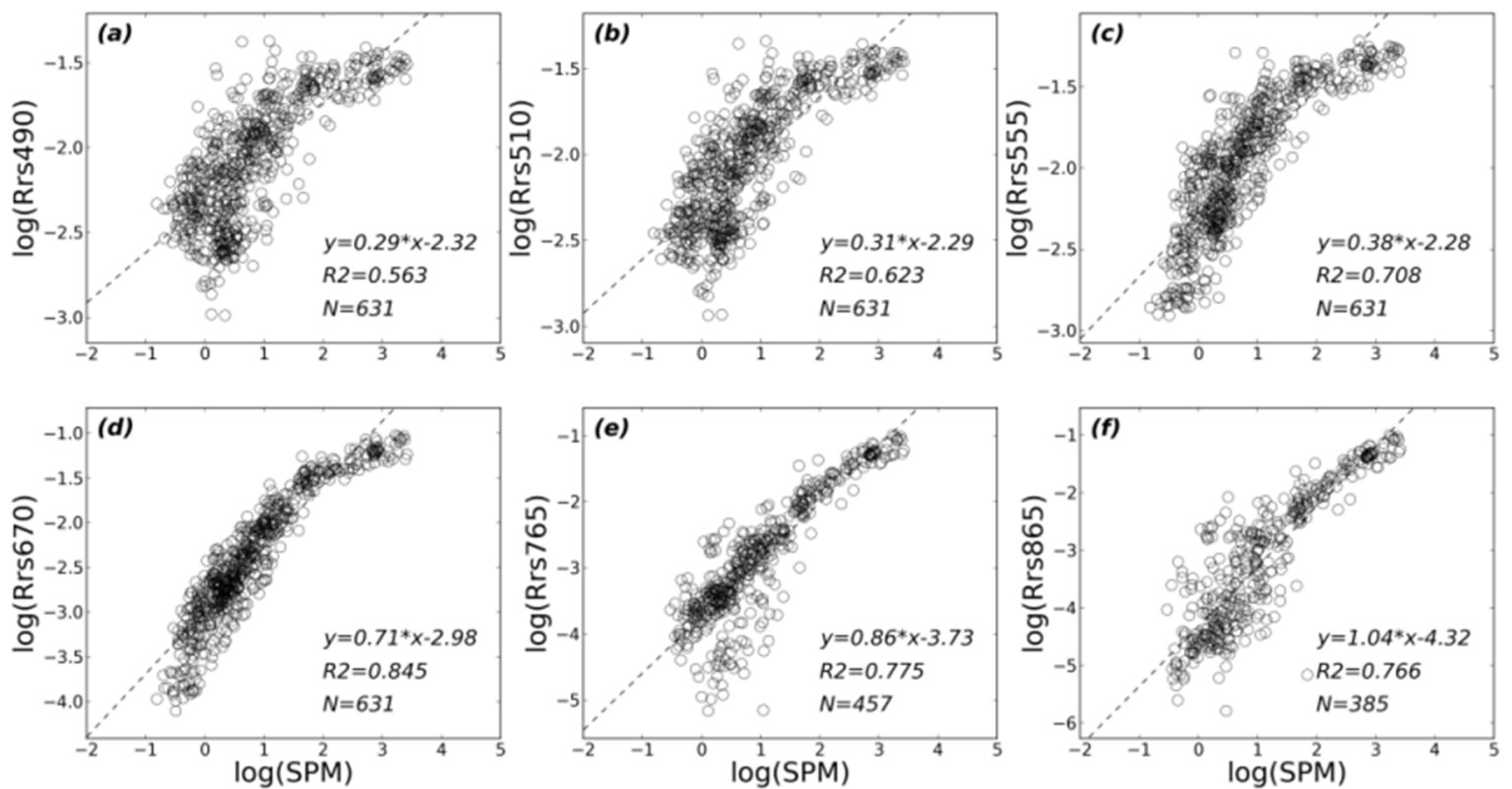

Figure 4. Variation of (a) $R_{\mathrm{rs}}(490)$; (b) $R_{\mathrm{rs}}(510) ;(\mathbf{c}) R_{\mathrm{rs}}(555)$; (d) $R_{\mathrm{rs}}(670) ;(\mathbf{e}) R_{\mathrm{rs}}(765)$; and (f) $R_{\mathrm{rs}}(865)$ as a function of $S P M$ in logarithmic space. The best linear regression fit is also plotted(dashed lines) in each panel. 

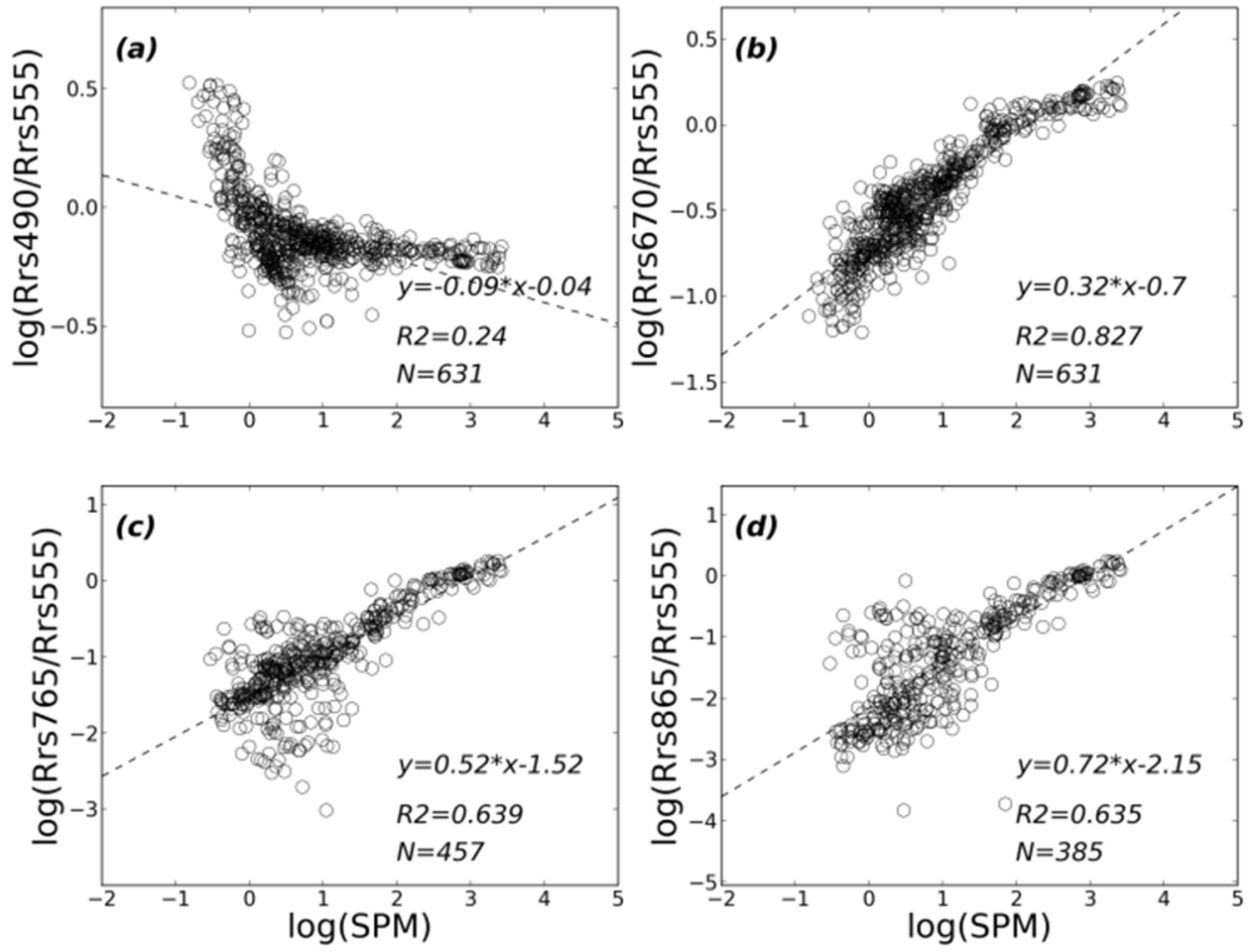

Figure 5. Variation of (a) $R_{\mathrm{rs}}(490) / R_{\mathrm{rs}}(555)$; (b) $R_{\mathrm{rs}}(670) / R_{\mathrm{rs}}(555) ; \quad$ (c) $R_{\mathrm{rs}}(765) / R_{\mathrm{rs}}(555)$; and (d) $R_{\mathrm{rs}}(865) / R_{\mathrm{rs}}(555)$ as a function of $S P M$ in logarithmic space. The best linear regression fit is also plotted(dashed line) in each panel.

\subsection{Performance of Historical Algorithms}

The equations of the four previously published algorithms selected for the present study are provided below for the SeaWiFS sensor.

(1) "Siswanto11" model (Siswanto et al. [24])

$$
\log _{10}(S P M)=0.649+25.623 \cdot \mathrm{X}_{1}+0.646 \cdot \mathrm{X}_{2}
$$

where $\mathrm{X}_{1}=R_{\mathrm{rs}}(555)+R_{\mathrm{rs}}(670), \mathrm{X}_{2}=R_{\mathrm{rs}}(490) / R_{\mathrm{rs}}(555)$.

(2) "Nechad10" model (Nechad et al. [26])

$$
S P M=384.11 \cdot \rho_{\mathrm{w}}(670) /\left(1-\rho_{\mathrm{w}}(670) / 0.1747\right)+1.44
$$

where $\rho_{\mathrm{w}}(670)=\pi \cdot R_{\mathrm{rs}}(670)$.

(3) "Doxaran03" model (Doxaran et al. [20])

$$
S P M=26.083 \times \exp (0.336 \mathrm{X})
$$

where $\mathrm{X}=R_{\mathrm{rs}}(865) / R_{\mathrm{rs}}(555)$. Note: this model was tuned for $S P M$ greater than $10 \mathrm{~g} \cdot \mathrm{m}^{-3}$.

(4) “Gohin05” model (Gohin et al. [29])

$$
S P M=n \operatorname{LvSPM}\left(n L_{\mathrm{w}}(555), n L_{\mathrm{w}}(670)\right)+234 C h l^{0.57}
$$

The look-up tables required for the $\mathrm{Chl}$ calculations and the coefficients required for the SPM calculations were provided by ACRI-HE with the agreement of IFREMER. 
The performance of these algorithms (Equations (9)-(12)), belonging to three different inverse approaches, are tested over the DS-T data set (Figure 6). The Siswanto11 model (Figure 6a), originally developed on $S P M$ values ranging between 0.04 and $340 \mathrm{~g} \cdot \mathrm{m}^{-3}$, provides the best retrieval accuracy with an RMSE_log of 0.33 over the whole SPM range considered in this study. This model tends, however, to underestimate SPM in relatively clear waters and, overestimate SPM in extremely turbid waters. The semi-analytical approach by Nechad et al. (2010) [26], Nechad10, also shows fairly good performance in SPM retrieval (RMSE_log=0.39), although with certain inherent limitations at low high SPM concentrations (Figure 6b), explaining the high Bias value of about $180 \%$. This algorithm, developed from $S P M$ values greater than $1.2 \mathrm{~g} \cdot \mathrm{m}^{-3}$, leads to over-estimation of $S P M$ at low concentration, due to the adoption of a constant offset (i.e., $1.44 \mathrm{~g} \cdot \mathrm{m}^{-3}$ for $\mathrm{B}^{\rho}$ ) which is reached for low $R_{\mathrm{rs}}(670)$ values. As soon as $\rho_{\mathrm{w}}(670)\left(=\pi \cdot R_{\mathrm{rs}}(670)\right)$ is greater than 0.1747 (a situation frequently encountered in highly to extremely turbid waters), negative $S P M$ values can also be generated (37 cases encountered in DS-T, not shown in Figure 6b). Such highly turbid waters were not considered in the calibration data set used by Nechad et al. (2010) [26] as their maximum SPM value reached $110.3 \mathrm{~g} \cdot \mathrm{m}^{-3}$ only. As expected, the Doxaran03 model appears to be fairly valuable for highly and extremely turbid waters (Figure 6c), but fails to perform well for low to medium SPM, as this model was originally developed for $S P M$ values ranging between 10 and $2000 \mathrm{~g} \cdot \mathrm{m}^{-3}$. It should also be noted that the number of valid $R_{\mathrm{rs}}(\lambda)$-SPM data points decreases significantly in DS-T when $R_{\mathrm{rs}}$ in the near-infrared range is used (e.g., $865 \mathrm{~nm}$ for SeaWiFS). The Gohin05 model (Gohin et al., 2005 [29]) provides relatively good retrieval of SPM for low to medium SPM concentrations, but is not able to accurately estimate SPM for concentration higher than about $50 \mathrm{~g} \cdot \mathrm{m}^{-3}$. The RMSE_log and bias values decrease from $0.376 \%$ and $127.3 \%$, respectively, when the whole data set is considered, to 0.363 and $118 \%$, when in situ SPM concentrations lower than $10 \mathrm{~g} \cdot \mathrm{m}^{-3}$ only are considered. Better SPM retrievals could certainly be achieved through, for instance, the improvement of the standard specific IOPs values used in the model, as already mentioned in Gohin (2011) [48]. Recently, the model has been improved thanks to a collaboration between IFREMER (F. Gohin) and the University of Bangor in Wales (D. Bowers). Unfortunately, this version is not available yet.
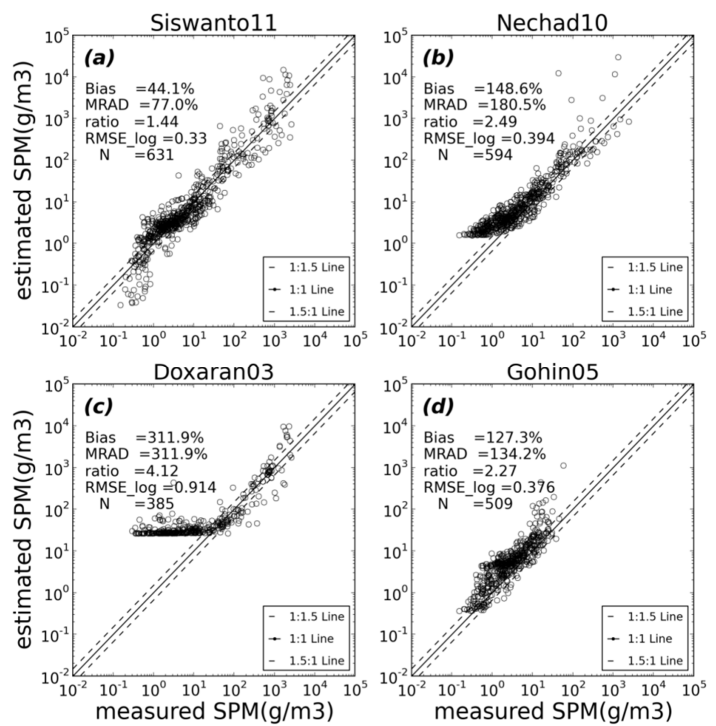

Figure 6. Comparison of the measured and inversed SPM values when the models of (a) Siswanto11, (b) Nechad10; (c) Doxaran03; and (d) Gohin05 are applied to the whole $R_{\mathrm{rs}}$ in situ data set. Solid and dashed lines represent the 1:1 and 1.5:1, 1:1.5 lines, respectively. The corresponding statistic indicators values are given in each panel. The number of data points are not always the same due to the limitation of the model (panel $(\mathbf{b}, \mathbf{d})$ ) or the unavailability of the input reflectance (panel $(\mathbf{c})$ ). 


\subsection{Performance of Tuned Algorithms}

The Siswanto-, Nechad-, and Doxaran-like models, as described in Equations (1)-(3), have been fitted to our development dataset (DS-D) in order to assess if these formulations can be used to retrieve $S P M$ spanning about four orders of magnitude. The new coefficients are provided in Table 3. Based on the relationship between $R_{\mathrm{rs}}$ band ratio and $S P M$, as shown in Figure 5, EA-BR model is re-designed to be in the form of $S P M \sim \mathrm{a}_{0} \cdot \mathrm{X}^{\mathrm{a} 1}$, where $\mathrm{X}$ is the $R_{\mathrm{rs}}$ band ratio. To avoid the cut-off situation encountered at low SPM range (Figure 6b), the offset parameter in Nechad-like model has been removed for this fitting exercise (i.e., $B^{\rho}=0$ in Equation (3)). Logically, the newly tuned models provide much better $S P M$ retrieval when compared to their original version, as emphasized by the statistics computed from the independent DS-V data set (Figure 7 and Table 4). For instance, the bias values decrease by about a factor of two between the original and fitted $E A-M B$ and $E A-B R$ models, and by a factor of four for the $S A A$ algorithm. This improvement is mainly due to the fact that original models were not developed for such a wide range in $S P M$ concentration. The relatively higher scatter observed around the 1:1 line for the $E A-B R$ model is related to the fact that this NIR based model was originally designed for medium to extremely turbid waters [20] for which signal to noise ratio is sufficiently high to draw a significant relationship between $S P M$ and $R_{\mathrm{rs}}$ at the considered wavelengths. The reversal feature explains the very high scatter observed for low to moderate SPM values thus confirming the inability of such NIR formulation to be used over the whole range of turbidity encountered in contrasted coastal waters (as already emphasized in Figures $4 \mathrm{f}$ and $5 \mathrm{~d}$ ).
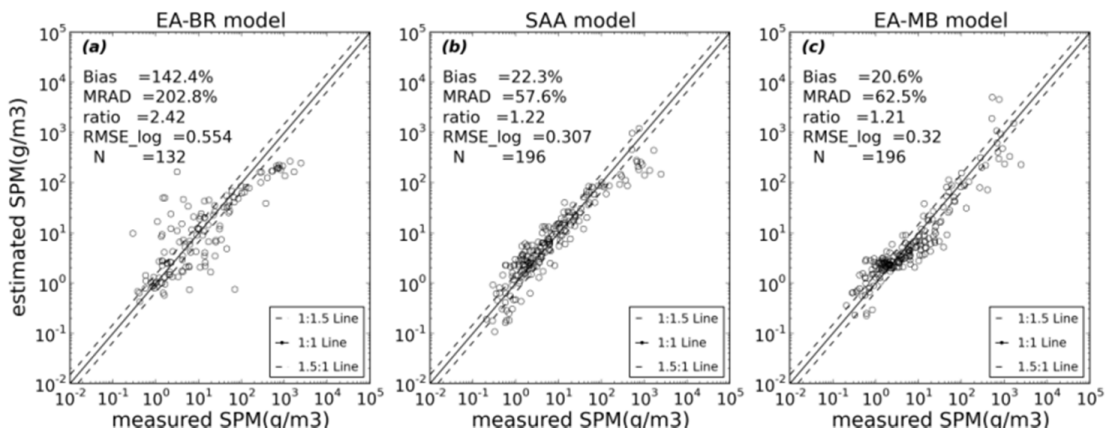

Figure 7. Comparison of the measured and inversed $S P M$ values when the (a) $E A-B R$; (b) $S A A$; and (c) $E A-M B$ models are applied to the DS-V in situ data set. Solid lines represent 1:1 line and dashed lines represent 1.5:1, 1:1.5 lines. The corresponding statistics indicators values are illustrated in each panel.

Table 4. Statistics of original (Equations (9)-(12)) and re-tuned models (Table 3 computed based on the validation sub-dataset (DS-V).

\begin{tabular}{cccccc}
\hline $\begin{array}{c}\text { Model } \\
\text { Reference }\end{array}$ & BIAS (\%) & MRAD (\%) & Ratio & RMSE_log & N \\
\hline Siswanto11 & 41.5 & 75.5 & 1.42 & 0.33 & 196 \\
Nechad10 & 98.7 & 130.1 & 1.99 & 0.37 & 186 \\
Doxaran03 & 208.8 & 208.8 & 3.09 & 0.91 & 132 \\
Gohin05 & 127.3 & 134.2 & 2.27 & 0.38 & 196 \\
EA-MB & 20.6 & 62.5 & 1.21 & 0.32 & 196 \\
EA-MB-L ${ }^{1}$ & $13.6(17.5)$ & $55.0(55.9)$ & $1.14(1.18)$ & $0.29(0.29)$ & 174 \\
EA-MB-H ${ }^{1}$ & $39.7(45.0)$ & $77.9(114.9)$ & $1.40(1.45)$ & $0.35(0.51)$ & 22 \\
SAA & 22.3 & 57.6 & 1.22 & 0.31 & 196 \\
SAA-L ${ }^{1}$ & $16.4(31.2)$ & $50.0(57.1)$ & $1.16(1.31)$ & $0.26(0.26)$ & 174 \\
SAA-H ${ }^{1}$ & $-15.4(-63.9)$ & $59.7(71.6)$ & $0.85(0.36)$ & $0.34(0.65)$ & 22 \\
EA-BR & 142.4 & 202.9 & 2.42 & 0.55 & 132 \\
EA-BR-L ${ }^{1}$ & $49.8(162.5)$ & $113,1(213.1)$ & $1.50(2.63)$ & $0.51(0.55)$ & 110 \\
EA-BR-H ${ }^{1}$ & $10.5(-65.8)$ & $35.5(65.8)$ & $1.11(0.34)$ & $0.22(0.58)$ & 22 \\
\hline
\end{tabular}

${ }_{1}^{1}$ numbers shown in parentheses in EA-MB-L, EA-MB-H, SAA-L, SAA-H, EA-BR-L and EA-BR-H rows represent corresponding statistics when EA-MB, SAA and/or EA-BR model are applied instead. 
Both $S A A$ (Figure $7 \mathrm{~b}$ ) and $E A-M B$ (Figure 7c) models perform relatively well for clear to medium turbid waters, but misestimate $S P M$ in highly to extremely turbid environments. For instance, the RMSE_log value increases from 0.32 to 0.51 when the $E A-M B$ model is applied for waters characterized by $S P M$ concentration higher than $100 \mathrm{~g} \cdot \mathrm{m}^{-3}$. In the same way, the RMSE_log value for the SAA model increases from 0.31 to 0.65 for $S P M$ concentration higher than $100 \mathrm{~g} \cdot \mathrm{m}^{-3}$. This feature clearly indicates that the SPM range should be explicitly taken into account within the inverse procedure development.

\subsection{New Formulations}

\subsubsection{Performance of SPM-Range Dependent Algorithms}

The previous results underline the inability of the three considered algorithms, even re-tuned on the DS-D data set, to properly deliver SPM over the whole SPM range considered. This can be more likely associated with the fact that the considered formulations and thus the hypotheses shaping these models are not able to fully capture the dynamics in the $R_{\mathrm{rs}}-S P M$ relationships over about four orders of magnitude in $S P M$ concentration. The latter results thus tend to indicate that turbidity conditions should be explicitly included in the inversion procedure if the aim is to deliver SPM concentration over extremely contrasting marine environments. In order to illustrate this feature, a first approach can consist in re-defining the three selected algorithms according to two different SPM ranges arbitrarily fixed to $S P M$ higher and lower than $100 \mathrm{~g} \cdot \mathrm{m}^{-3}$. For each of the three models $(S A A, E A-M B$, and $E A-B R)$, two distinct sub-models have been developed considering low $(\mathrm{L})$ and high $(\mathrm{H}) S P M$ conditions. Each sub-model is derived by retuning the coefficients appearing in Equations (1)-(3), in the two SPM ranges based on the DS-D dataset. Note the modification of $E A-B R$ model (see Section 3.3). Even though $100 \mathrm{~g} \cdot \mathrm{m}^{-3}$ seems arbitrary, it should represent a credible threshold from a qualitative perspective (see Figure 7). A proper selection of an optical segment index will be specifically assessed in the following section.

The $S P M$-range dependent algorithms allow $S P M$ to be retrieved with a much better accuracy for the former two considered approaches (i.e., $S A A$ and $E A-B R$ models, Figure 8 and Table 4). For instance, the RMSE_log value decreases from 0.65 to 0.34 for $S A A$ and from 0.58 to 0.22 for $E A-B R$, for $S P M$ concentrations higher than $100 \mathrm{~g} \cdot \mathrm{m}^{-3}$. The same results are observed, to a lesser extent, for the lower SPM range (Table 4). For instance, the RMSE_log value decreases from 0.36 to 0.26 when the $S A A$ and $S A A-L$ models are used, respectively, in this lower $S P M$ range $\left(S P M<100 \mathrm{~g} \cdot \mathrm{m}^{-3}\right)$.Note that the $E A-M B$ model is not considered in Figure 8 because the $S P M$-range dependent approach was not able to give better results than the former $E A-M B$ model.
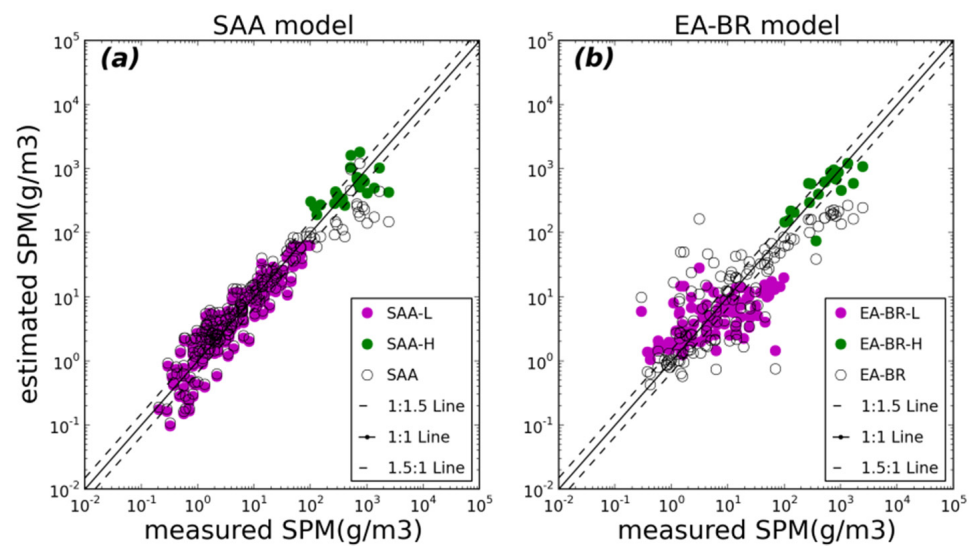

Figure 8. Comparison of the measured and inversed $S P M$ values when (a) $S A A$; and (b) EA-BR models are applied to the DS-V in situ data set. Open, green, and pink circles stand for the version of each model developed over the Whole, High and Low SPM ranges, respectively (see Table 3). Solid lines represent 1:1 line and dashed lines represent 1.5:1, 1:1.5 lines. 


\subsubsection{Generic Semi-Analytical Model}

Because of its physical foundation, as well as its slightly better performance compared to the two empirical approaches considered here, the generic algorithm is developed based on the $S A A$ formulation only. As previously emphasized, SPM concentration can be retrieved with a substantially improved accuracy when clear to medium turbid waters and highly to extremely turbid waters are considered separately. The applicability of such an inversion algorithm is, however, conditioned by the possibility to define a switching point between these latter two turbidity conditions based on an optical criterion. Among the several optical switching indexes possible (e.g., single band or band ratio based threshold), the remote-sensing reflectance, $R_{\mathrm{rs}}$, in the red domain (i.e., $670 \mathrm{~nm}$ for SeaWiFS) was found to be the most valuable proxy for defining on a pixel basis low-medium or high turbidity environment, and thus for choosing the proper SPM inversion algorithm. However, considering that remote-sensing reflectance at red wavelength may saturate for the extremely high SPM range, while one algorithm has been developed for the low-medium SPM range (named $S A A-L$ ), two different algorithms have to then be tested for the higher $S P M$ range. The first one (named $S A A-H R$ ) used $R_{\mathrm{rs}}$ in the red, while the second one (named $S A A-H N I R$ ), which should be more sensitive to very high SPM concentrations, used $R_{\mathrm{rs}}$ in the near-infrared waveband (i.e., $765 \mathrm{~nm}$ for SeaWiFS).

In order to determine an appropriate threshold based on a radiometric index (e.g., single band or band ratio), two situations are considered separately. One heuristic way consists in considering an increasing threshold value, then to re-formulate model coefficients for each critical value, and compare the evolution in the performance of the SPM inversion for the two sub-validation datasets as defined by the chosen threshold. This first approach is defined as the "lower critical value". The same procedure can be applied to the other direction considering an index that is confined to be not much greater than a gradually decreasing critical value ("upper critical value"). The determination of both optimal values is assessed from the validation statistics and requires a sufficient number of records to ensure the test's representativeness. Both single band and band ratios can be considered as a possible index. However, single band remote-sensing reflectance can be assumed to represent a more suitable index since it correlates with $S P M$ in a more straightforward way than band ratio does. By iterating lower and upper critical values of $R_{\mathrm{rs}}(670)$, for the case of the SeaWiFS sensor, 0.03 and $0.04 \mathrm{sr}^{-1}$ have been determined from the current data set as optimal lower and upper critical values, respectively. The coefficients for these three algorithms using the DS-D data set are provided in Table 5. Table 6 gives statistics when these SPM-dependent models are validated using DS-V.

The consideration of two distinct algorithms based on the relative turbidity of the water masses for remote sensing application might induce an artificial spatial pattern in the SPM distribution. To prevent such possible irregularity, a smoothing procedure has thus to be applied especially for regions intermediary between medium and high turbid situations. The smoothing method adopted consists in defining a weighting function of the low-medium and high SPM algorithms. In practice, two weights $\left(\mathrm{W}_{\mathrm{L}}\right.$ and $\mathrm{W}_{\mathrm{H}}$ for low and high SPM conditions, respectively) have been defined as follows:

$$
\begin{gathered}
\mathrm{W}_{\mathrm{L}}=\left\{\begin{array}{c}
1, \text { if } R_{\mathrm{rs}}\left(\lambda_{0}\right) \leqslant 0.03 \mathrm{sr}^{-1} \\
0, \text { if } R_{\mathrm{rs}}\left(\lambda_{0}\right) \geqslant 0.04 \mathrm{sr}^{-1} \\
\mathrm{~W}_{\mathrm{H}}=\left\{\begin{array}{c}
0, \text { if } R_{\mathrm{rs}}\left(\lambda_{0}\right) \leqslant 0.03 \mathrm{sr}^{-1} \\
1, \text { if } R_{\mathrm{rs}}\left(\lambda_{0}\right) \geqslant 0.04 \mathrm{sr}^{-1} \\
\log _{10}(0.04)-\log _{10}\left[R_{\mathrm{rs}}\left(\lambda_{0}\right)\right], \text { otherwise }
\end{array}\right. \\
\log _{10}\left[R_{\mathrm{rs}}\left(\lambda_{0}\right)\right]-\log _{10}(0.03), \text { otherwise }
\end{array}\right.
\end{gathered}
$$

where $\lambda_{0}$ is $670 \mathrm{~nm}, 667 \mathrm{~nm}, 667 \mathrm{~nm}, 665 \mathrm{~nm}, 671 \mathrm{~nm}$ and $655 \mathrm{~nm}$ for SeaWiFS, MODIS-A, MODIS-T, MERIS/MSI/OLCI, VIIRS and OLI, respectively. The weighted SPM is then computed as:

$$
S P M=\frac{\mathrm{W}_{\mathrm{L}} \cdot S P M_{\mathrm{L}}+\mathrm{W}_{\mathrm{H}} \cdot S P M_{\mathrm{H}}}{\mathrm{W}_{\mathrm{L}}+\mathrm{W}_{\mathrm{H}}}
$$


Where $S P M_{L}$ and $S P M_{H}$ are estimated by models calibrated for low turbidity (i.e., $S A A-L$ ) and high turbidity(i.e., $S A A-H R$ or $S A A-H N I R)$ environments, respectively.

Table 5. Coefficients of the SPM-dependent models developed on DS-D for various Ocean Color sensors.

\begin{tabular}{cccccc}
\hline $\begin{array}{c}\text { Model } \\
\text { Index }\end{array}$ & Sensor Name & $\begin{array}{c}\text { WavebandsInvolved } \\
(\mathbf{n m})^{\mathbf{1}}\end{array}$ & $\begin{array}{c}\text { Switching } \\
\text { Threshold }\end{array}$ & $\begin{array}{c}\text { Retuned } \\
\text { Coefficients }{ }^{2}\end{array}$ & N \\
\hline \multirow{5}{*}{ SAA-L } & SeaWiFS & 670 & $R_{\mathrm{rs}}(670) \leqslant 0.03$ & $391.161,0.5$ & 366 \\
& MODIS-A & 667 & $R_{\mathrm{rs}}(667) \leqslant 0.03$ & $404.400,0.5$ & 366 \\
& MODIS-T & 667 & $R_{\mathrm{rs}}(667) \leqslant 0.03$ & $404.400,0.5$ & 366 \\
& MERIS/OLCI/MSI & 665 & $R_{\mathrm{rs}}(665) \leqslant 0.03$ & $396.005,0.5$ & 365 \\
& VIIRS & 671 & $R_{\mathrm{rs}}(671) \leqslant 0.03$ & $389.471,0.5$ & 366 \\
& OLI & 655 & $R_{\mathrm{rs}}(655) \leqslant 0.03$ & $346.353,0.5$ & 362 \\
\hline \multirow{5}{*}{ SAA-HR } & SeaWiFS & 670 & $R_{\mathrm{rs}}(670) \geqslant 0.04$ & $1336.584,0.3864$ & 49 \\
& MODIS-A & 667 & $R_{\mathrm{rs}}(667 \geqslant 0.04$ & $1214.669,0.3394$ & 46 \\
& MODIS-T & 667 & $R_{\mathrm{rs}}(667) \geqslant 0.04$ & $1214.669,0.3394$ & 46 \\
& MERIS/OLCI/MSI & 665 & $R_{\mathrm{rs}}(665) \geqslant 0.04$ & $1208.481,0.3375$ & 46 \\
& VIIRS & 671 & $R_{\mathrm{rs}}(671) \geqslant 0.04$ & $1234.599,0.3439$ & 47 \\
& OLI ${ }^{3}$ & 655 & $R_{\mathrm{rs}}(655) \geqslant 0.045$ & $1221.390,0.3329$ & 38 \\
\hline \multirow{2}{*}{ SAA-NIR } & SeaWiFS & 765 & $R_{\mathrm{rs}}(670) \geqslant 0.04$ & $2245.985,0.4168$ & 49 \\
& MODIS-A & 748 & $R_{\mathrm{rs}}(667 \geqslant 0.04$ & $2201.029,0.3975$ & 46 \\
& MODIS-T & 748 & $R_{\mathrm{rs}}(667) \geqslant 0.04$ & $2201.029,0.3975$ & 46 \\
& MERIS/OLCI/MSI & 753 & $R_{\mathrm{rs}}(665) \geqslant 0.04$ & $2220.066,0.4029$ & 46 \\
& VIIRS & 745 & $R_{\mathrm{rs}}(671) \geqslant 0.04$ & $2198.675,0.3951$ & 47 \\
\hline
\end{tabular}

\footnotetext{
${ }^{1}$ Selected wavebands (i.e., $\lambda_{0}$ ) in Equation (2). ${ }^{2}$ Retuned coefficients for $\mathrm{A}^{\rho}$ and $\mathrm{C}^{\rho}$ in Equation (2), respectively, while $\mathrm{B}^{\rho}=0 ;{ }^{3}$ For OLI (center wavelength $=655 \mathrm{~nm}$ ), $0.045 \mathrm{sr}^{-1}$ works better as upper switching point than $0.04 \mathrm{sr}^{-1} ;{ }^{4} \mathrm{MSI}$ and OLI do not have wavebands in the near-infrared domain (e.g., $765 \mathrm{~nm}$ for SeaWiFS).
}

These two sets of algorithms, that is $S A A-L$ combined with $S A A-H R$, and $S A A-L$ combined with $S A A-H N I R$, are applied to the in situ data set DS-V to evaluate their respective performance (Table 7). Compared to the performances reached by the $S A A$ algorithm developed over the whole $S P M$ range, the application of the SPM-range dependent algorithms provides a much better SPM retrieval. For instance, the RMSE_log value calculated over the whole SPM range is 0.31 for $S A A$, 0.27 for combination of (SAA-L, SAA-HR), and 0.26 for that of (SAA-L, SAA-HNIR), respectively (Table 7). Compared to $S A A$ (Table 4), the Bias value decreases by a factor of 1.27 and 1.44 for $(S A A-L, S A A-H R)$, and $(S A A-L, S A A-H N I R)$, respectively. At high turbid waters (e.g., $\left.R_{\mathrm{rs}}(670) \geqslant 0.04 \mathrm{sr}^{-1}\right)$, the $S A A-H N I R\left(R M S E \_l o g=0.28 ; M R A D=40.7 \%\right)$ presents slightly better $S P M$ retrieval than $S A A-H R\left(R M S E \_l o g=0.33 ; M R A D=52.7 \%\right)$. This difference observed between $S A A-H R$ and SAA-HNIR is simply explained by the tighter linear behavior between $R_{\mathrm{rs}}$ and SPM in the near-infrared than in the red part of the spectrum (Figure 4). The latter general statistics vary only slightly from one sensor to another (i.e., SeaWiFS, MODIS, MERIS VIIRS, OLCI, OLI, MSI) demonstrating the great potential applicability of using such combined approaches (Table 7).

Further, the application of such a smoothing procedure (Equations (13)-(15)) on MERIS data confirms that the switching and smoothing method proposed here allows the prevention of any artificial discontinuity in the SPM coastal to offshore dispersion gradient (see Figure 9). The SPM retrieval follows the same spatial course as the one described by $R_{\mathrm{rs}}(665)$, with no obvious discontinuity observed at the switching domain, that is for $R_{\mathrm{rs}}(665)$ values between 0.03 and $0.04 \mathrm{sr}^{-1}$. Besides, the estimation of concentration as high as $1300 \mathrm{~g} \cdot \mathrm{m}^{-3}$ (around the latitude $48.8^{\circ} \mathrm{S}$ in Figure $8 \mathrm{~b}$ ) clearly stresses that the use of the remote sensing reflectance in the red, combined with this switching method, is totally adapted to very turbid waters. 
Table 6. Validation of SPM-dependent models on DS-V.

\begin{tabular}{|c|c|c|c|c|c|c|c|}
\hline $\begin{array}{l}\text { Model } \\
\text { Name }\end{array}$ & Sensor Name & $\begin{array}{l}\text { Switching } \\
\text { Threshold }\end{array}$ & Bias (\%) & $\begin{array}{c}\text { MRAD } \\
(\%)\end{array}$ & Ratio & RMSE_log & $\mathbf{N}$ \\
\hline \multirow{6}{*}{ SAA-L } & SeaWiFS & $R_{\mathrm{rs}}(670) \leqslant 0.03$ & 17.8 & 51.4 & 1.18 & 0.263 & 166 \\
\hline & MODIS-A & $R_{\mathrm{rs}}(667) \leqslant 0.03$ & 18.4 & 51.9 & 1.18 & 0.263 & 166 \\
\hline & MODIS-T & $R_{\mathrm{rs}}(667) \leqslant 0.03$ & 18.4 & 51.9 & 1.18 & 0.263 & 166 \\
\hline & MERIS/OLCI/MSI & $R_{\mathrm{rs}}(665) \leqslant 0.03$ & 18.8 & 52.5 & 1.19 & 0.264 & 165 \\
\hline & VIIRS & $R_{\mathrm{rs}}(671) \leqslant 0.03$ & 17.5 & 51.2 & 1.18 & 0.263 & 166 \\
\hline & OLI & $R_{\mathrm{rs}}(655) \leqslant 0.03$ & 15.5 & 50.6 & 1.16 & 0.261 & 166 \\
\hline \multirow{6}{*}{ SAA-HR } & SeaWiFS & $R_{\mathrm{rs}}(670) \geqslant 0.04$ & -0.4 & 52.1 & 1.00 & 0.329 & 18 \\
\hline & MODIS-A & $R_{\mathrm{rs}}(667 \geqslant 0.04$ & 9.7 & 59.0 & 1.10 & 0.340 & 18 \\
\hline & MODIS-T & $R_{\mathrm{rs}}(667) \geqslant 0.04$ & 9.7 & 59.0 & 1.10 & 0.340 & 18 \\
\hline & MERIS/OLCI/MSI & $R_{\mathrm{rs}}(665) \geqslant 0.04$ & 10.0 & 59.4 & 1.10 & 0.342 & 18 \\
\hline & VIIRS & $R_{\mathrm{rs}}(671) \geqslant 0.04$ & 9.7 & 58.4 & 1.10 & 0.337 & 18 \\
\hline & $\mathrm{OLI}^{1}$ & $R_{\mathrm{rs}}(655) \geqslant 0.045$ & 2.5 & 56.3 & 1.03 & 0.347 & 16 \\
\hline \multirow{6}{*}{ SAA-HNIR } & SeaWiFS & $R_{\mathrm{rs}}(670) \geqslant 0.04$ & 06.5 & 40.7 & 0.94 & 0.277 & 18 \\
\hline & MODIS-A & $R_{\mathrm{rs}}(667 \geqslant 0.04$ & -5.6 & 41.7 & 0.94 & 0.282 & 18 \\
\hline & MODIS-T & $R_{\mathrm{rs}}(667) \geqslant 0.04$ & -5.6 & 41.7 & 0.94 & 0.282 & 18 \\
\hline & MERIS/OLCI & $R_{\mathrm{rs}}(665) \geqslant 0.04$ & -5.9 & 41.4 & 0.94 & 0.281 & 18 \\
\hline & VIIRS & $R_{\mathrm{rs}}(671) \geqslant 0.04$ & -5.2 & 42.0 & 0.95 & 0.282 & 18 \\
\hline & OLI & - & - & - & - & - & - \\
\hline
\end{tabular}

${ }^{1}$ For OLI (center wavelength $\left.=655 \mathrm{~nm}\right), 0.045 \mathrm{sr}^{-1}$ works more effectively as an upper switching point than $0.04 \mathrm{sr}^{-1}$.

Table 7. Performance of combined SPM-dependent models for various ocean color sensors on DS-V.

\begin{tabular}{|c|c|c|c|c|c|c|}
\hline Model Name & Sensor Name & RPD (\%) & APD (\%) & Ratio & RMSE_log & $\mathbf{N}$ \\
\hline \multirow{2}{*}{ Switch_1 } & SeaWiFS & 19.6 & 54.5 & 1.20 & 0.274 & 196 \\
\hline & MODIS-A & 21.1 & 55.5 & 1.21 & 0.275 & 196 \\
\hline \multirow{2}{*}{ SAA-L+ } & MODIS-T & 21.1 & 55.5 & 1.21 & 0.275 & 196 \\
\hline & MERIS/OLCI/MSI & 21.5 & 56.0 & 1.22 & 0.275 & 196 \\
\hline \multirow{2}{*}{ SAA-HR } & VIIRS & 20.1 & 54.7 & 1.20 & 0.274 & 196 \\
\hline & OLI & 20.3 & 54.2 & 1.20 & 0.270 & 196 \\
\hline \multirow{2}{*}{ Switch_2 } & SeaWiFS & 15.5 & 50.1 & 1.16 & 0.261 & 196 \\
\hline & MODIS-A & 16.3 & 50.7 & 1.16 & 0.262 & 196 \\
\hline \multirow{2}{*}{ SAA-L+ } & MODIS-T & 16.3 & 50.7 & 1.16 & 0.262 & 196 \\
\hline & MERIS/OLCI/MSI ${ }^{1}$ & 16.5 & 51.0 & 1.17 & 0.262 & 196 \\
\hline \multirow{2}{*}{ SAA-HNIR } & VIIRS & 15.4 & 50.0 & 1.15 & 0.261 & 196 \\
\hline & OLI $^{1}$ & - & - & - & - & - \\
\hline
\end{tabular}

${ }^{1}$ MSI and OLI do not have wavebands in the near-infrared spectral domain comparable to that of other Ocean Color sensors (e.g., $765 \mathrm{~nm}$ for SeaWiFS).
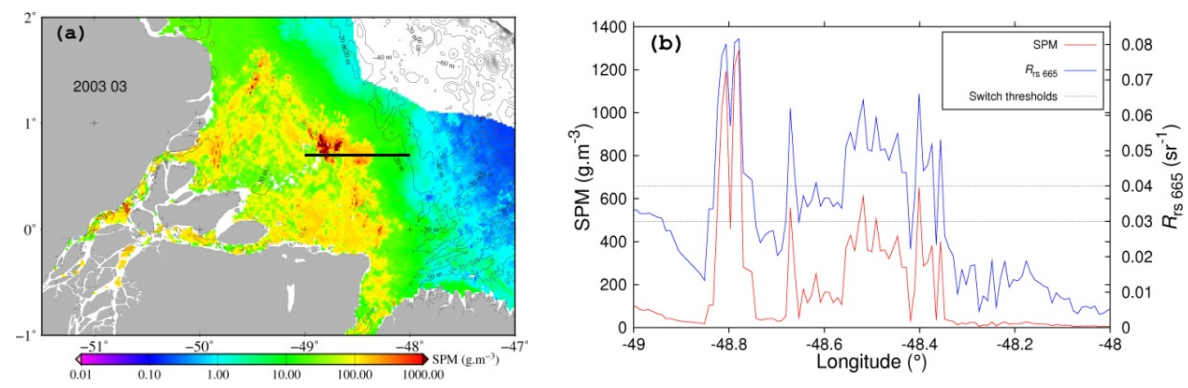

Figure 9. (a) Monthly SPM image (March 2003) obtained by applying the SAA generic algorithm (Equations (13)-(15)) to the monthly mean MERIS data collected over the Amazon coastal waters. The black line on the image represents the location of the transect over which the SPM and $R_{\mathrm{rs}}(665)$ data are extracted; (b) Spatial evolution of $S P M$ and $R_{\mathrm{rs}}(665)$ along the transect. The two dashed lines indicate the adopted switching thresholds. 


\section{Sensitivity Analysis}

\subsection{Sensitivity of the SPM Retrieval Accuracy to the $b_{b p} / S P M$ Variability}

This section aims at verifying whether the accuracy of the SPM retrieval is partly linked to the $S P M$ mass-specific backscattering coefficient $\left(b_{\mathrm{bp}}^{*}\right)$ variability. This exercise is performed for the SAA algorithm only. From the SPM retrieved values using this latter algorithm (Figure 10), three subsets of data were generated based on the ratio value of estimated to measured SPM as computed from DS-IOP-V. The first group $\left(\mathrm{D}_{\text {over }}\right)$ gathers data with a ratio value greater than 1.5 (significantly over-estimated SPM), while the second group $\left(\mathrm{D}_{\text {under }}\right)$ concerns data with a ratio lower than $1 / 1.5$ (significantly under-estimated $S P M$ ). The remaining data points, which represent the best estimated SPM values, lie in the third group $\left(\mathrm{D}_{\text {best }}\right)$. The mean $b_{\mathrm{bp}}^{*}(488)$ calculated for the data points gathered into $D_{\text {over }}\left(0.025 \pm 0.011 \mathrm{~m}^{2} \cdot \mathrm{g}^{-1}\right)$ is about two times higher than the one calculated over $D_{\text {under }}\left(0.011 \pm 0.004 \mathrm{~m}^{2} \cdot \mathrm{g}^{-1}\right)$ (Figure 10a). Interestingly, the remaining data points for which the estimation of SPM from the SAA algorithm is relatively good, present intermediate $b_{\mathrm{bp}}^{*}(488)$ value $\left(0.013 \pm 0.006 \mathrm{~m}^{2} \cdot \mathrm{g}^{-1}\right)$. This result strongly emphasizes that the accuracy in the SPM retrieval is tightly linked to the bulk particulate matter chemical composition and size. Higher $b_{\mathrm{bp}}^{*}(488)$ values are, for instance, generally observed for waters where the particulate fraction is dominated by the presence of mineral particles [37]. These results regarding the impact of the specific backscattering coefficient on the SPM retrieval are in line with the study of Binding et al. (2005) [51] performed on moderately turbid waters.
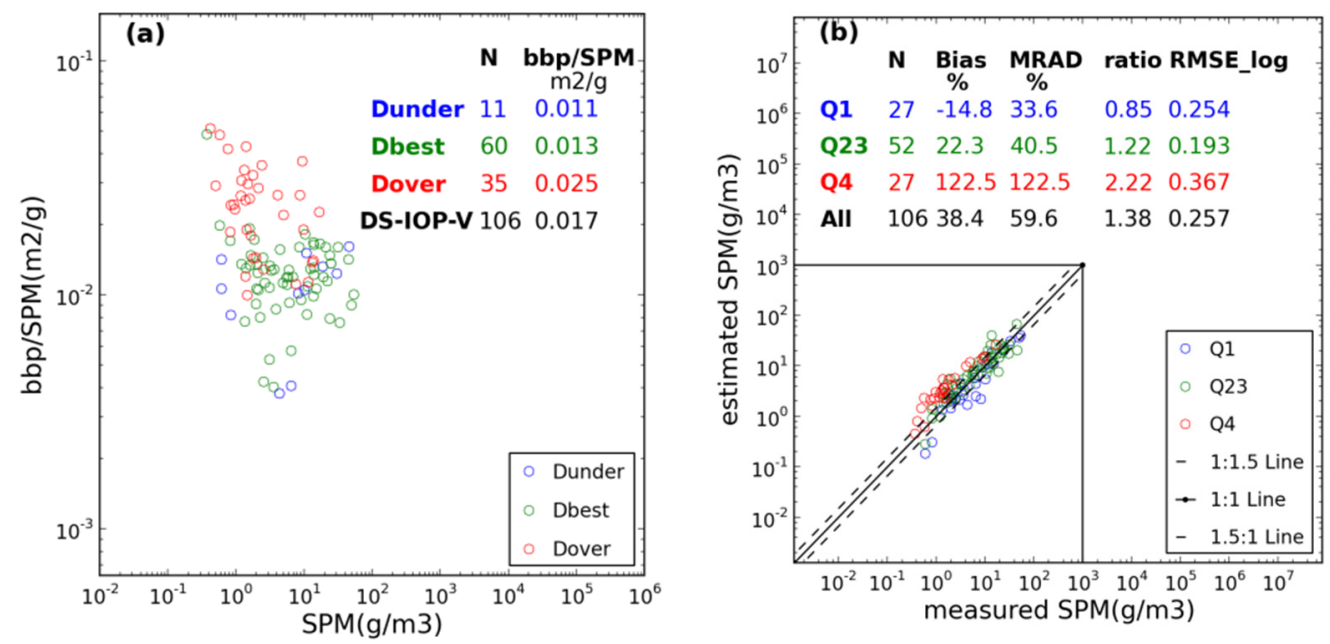

Figure 10. (a) Repartition of the $b_{\mathrm{bp}} / S P M$ values when SPM is over estimated (red dots), under-estimated (green dots), well estimated (see text); (b) Comparison of the measured and inversed-SAA SPM values. The points belonging to each of the four defined quartile are represented as indicated. The values of the statistical indicators are provided for each quartile. Solid lines represent 1:1 line and dashed lines represent 1.5:1, 1:1.5 lines.

The actual effect of the variability in $b_{\mathrm{bp}}^{*}$ on $S P M$ retrieval has been examined quantitatively. For that purpose, DS-IOP-V is re-organized into three sub-groups based on quartiles in terms of $b_{\mathrm{bp}}^{*}$ following the Loisel et al.'s (2010) approach [45]. The first quartile cuts off the lowest $25 \%$ of the $b_{\mathrm{bp}}^{*}$ data. The corresponding sub data set is named DS-IOP-VQ1. The third quartile cuts off the highest $25 \%$ into a sub-dataset named DS-IOP-VQ4. The remaining goes into DS-IOP-VQ23. The SPM-retrieval accuracy through the use of SAA significantly differs between DS-IOP-VQ1 and DS-IOP-VQ4 (Figure 10b). For instance, a negative bias of $-21.1 \%$ is observed for the data point of DS-IOP-VQ1, while a positive bias of $74.8 \%$ is observed for DS-IOP-VQ4. 
In the $S A A$ model (see Section 2.2), $C^{\rho}$ is related to $b_{\mathrm{bp}}^{*}$ through $C^{\rho}=\gamma \cdot b_{\mathrm{bp}}^{*} /\left(a_{\mathrm{p}}^{*}+b_{\mathrm{bp}}^{*}\right)$, with $a_{\mathrm{p}}^{*}$ and $\gamma$ held constant and taken from Nechad et al. (2010) [26], while $\mathrm{A}^{\rho}$ (see Equation (3)) is determined through a non-linear least square fit. A general model (called "M0") was obtained considering the whole DS-IOP-D data set. Similarly, for each quartile-based sub-group of DS-IOP-D (i.e., DS-IOP-DQ1, DS-IOP-DQ23 and DS-IOP-DQ4, the same division is applied to DS-IOP-V), new sets of $\mathrm{C}^{\rho}$ and $\mathrm{A}^{\rho}$ were calculated leading to three associated models ("M1", "M23", and "M4"). The performance of these three models was evaluated over the DS-IOP-V data set (Figure 11). A substantial improvement is observed over the DS-IOP-VQ1 and DS-IOP-VQ4 data sets when the model parameters are adjusted regarding to their mean $b_{b p}^{*}$ values. For instance, the RMSE_log value decreases from 0.318 (with "M0" model) to 0.264 (with "M1" model) for DS-IOP-VQ1. In the same way, the RMSE_log value decreases from 0.277 (with" M0" model) to 0.172 (with "M4" model) for DS-IOP-VQ4. Besides, the SPM retrieval accuracy remains equivalent between "M0" and "M23" model. The same result has been observed for the other algorithms presented here (not shown). These results strongly emphasize that implicit knowledge on the $b_{b p}^{*}$ values, combined with appropriated SAA algorithms, would significantly improve the SPM assessment from the marine reflectance (at least for the SPM range tested here).
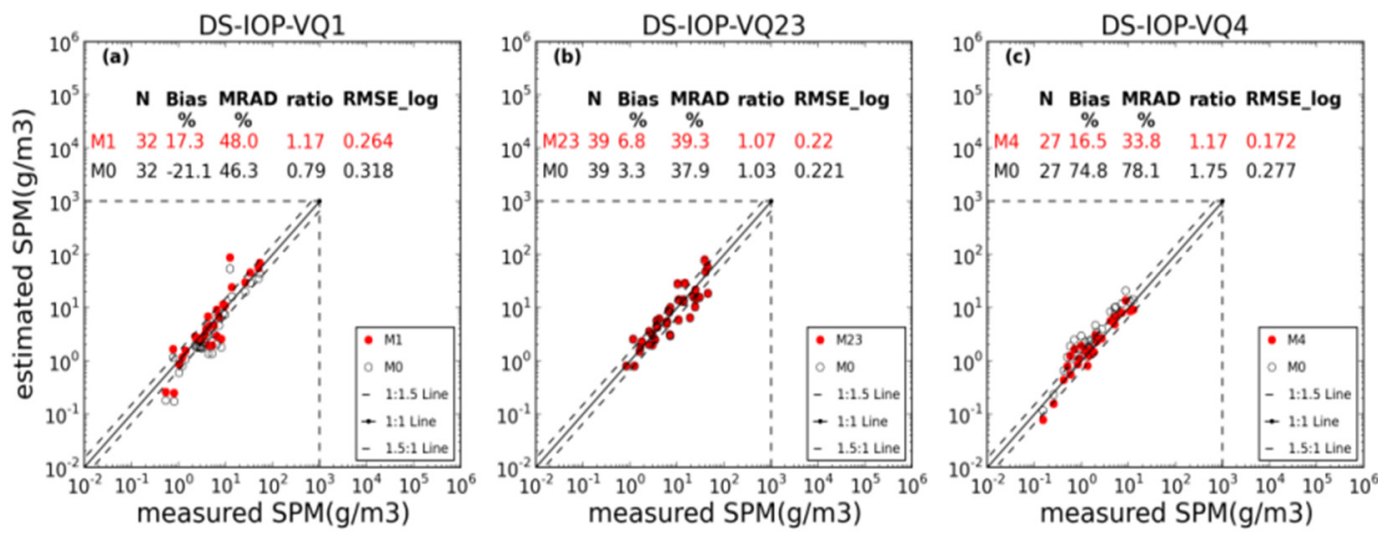

Figure 11. Comparison of the measured and inversed $S P M$ by $S A A$ model for the data points of the $Q 1, Q 23$, and $Q 4$ quartiles. In each panel, black circles represent the $S P M$ values estimated using the averaged $C^{\rho}$ value over $D S-I O P-D$, and red circles represent those estimated from the averaged $C^{\rho}$ value over each quartile-averaged. The values of the statistical indicators are provided for each quartile. Solid line represent 1:1 line and dashed lines represent 1.5:1, 1:1.5 lines. (a) DS-IOP-VQ1;

(b) DS-IOP-VQ23; (c) DS-IOP-VQ4.

\subsection{Effect of Rrs Uncertainties on the SPM Retrieval}

The impact $R_{\mathrm{rs}}$ retrieval errors, mainly caused by uncertainties in the atmospheric correction procedure, on the $S P M$ retrieval accuracy was examined for the $E A-B R, S A A$ and $E A-M B$ models. For that purpose, relative error budget for the $R_{\mathrm{rs}}$ signal in the blue, green, and red domains was assumed to be normally distributed with mean (and standard deviation) values of $-0.2 \%(0.05 \%),-3 \%$ $(0.6 \%)$, and $-25 \%(5 \%)$, respectively. The latter mean values were fixed according to the results of the match-up exercise performed for the MODIS-aqua sensor using the NASA standard NIR-atmospheric correction scheme [52]. Statistically, $R_{\mathrm{rs}}$ uncertainties causes $S P M$ to be under-estimated for all cases, with RMSE_log increases from $0.554,0.307$, and 0.320 to $0.657,0.363$, and 0.374 for $E A-B R, S A A$ and $E A-M B$ models, respectively.

\subsection{Impact of the Spectral Response Function of Each Ocean Color Sensors}

The models developed in this study were developed from coinciding in situ $\mathrm{R}_{\mathrm{rs}}$ and SPM measurements. Specifically, $\mathrm{R}_{\mathrm{rs}}$ were calculated over a $10 \mathrm{~nm}$-bandwidth whenever possible i.e., for hyper-spectral measurements. However, in order to check for the ability of the proposed models 
to be applied to the different ocean color sensors available, $R_{\mathrm{rs}}$ data should be weighted according to the corresponding Spectral Response Functions, SRF, for each ocean color sensor [53]. This step, which is recommended in the case of high spatial resolution sensors with large spectral bandwidths such as SPOT [54] or Landsat ETM+ [11], is here tested with narrow bands of ocean color sensors. Comparison between these two sets of $R_{\mathrm{rs}}$ (i.e., averaged over $10 \mathrm{~nm}$, or using the appropriated SRF) is performed for the selected bands of SeaWiFS, MODIS-Aqua, MODIS-Terra, MERIS, VIIRS and OLI sensors (Figure 12), respectively. Except for several points in the near-infrared spectral region which significantly deviate from the 1:1 line, $R_{\mathrm{rs}}$ data calculated by the two methods are in perfect agreement (Figure 12). This indicates that previously re-tuned models can be applied without any change to ocean color remote sensing data provided by these sensors. In the near-infrared domain, SRF usually covers a large band width (i.e., $40 \mathrm{~nm}$ or more), making it more vulnerable to abnormal values. Therefore, one should be very careful about the quality control for this part of the spectrum.
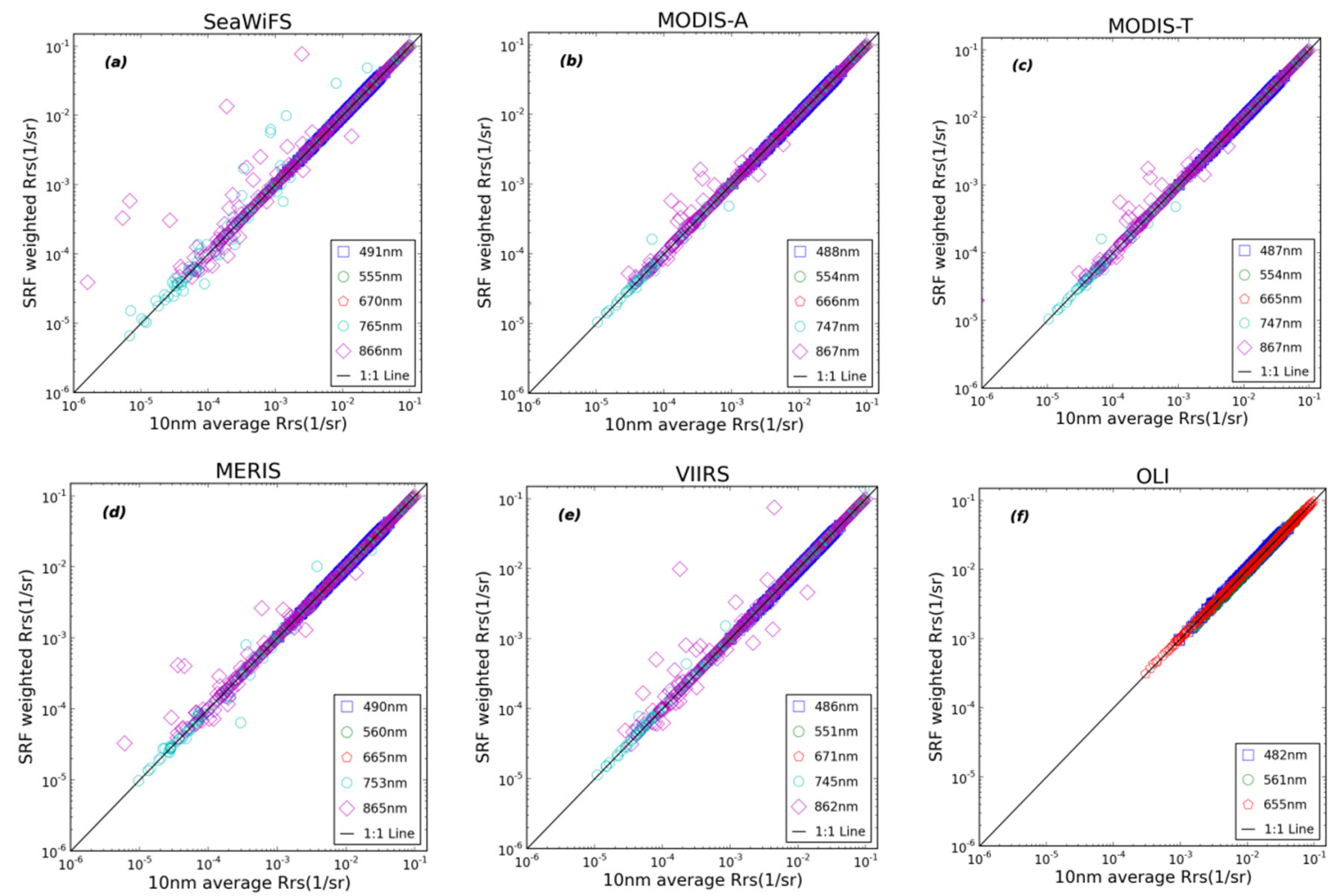

Figure 12. Comparison between the $R_{\mathrm{rs}}$ values averaged over a $10 \mathrm{~nm}$-bandwidth, and those calculated using the Spectral Response Functions for the (a) SeaWiFS; (b) MODIS-Aqua; (c) MODIS-Terra; (d) MERIS (e) VIIRS and (f) OLI sensors for visible and near-infrared channels, as indicated. Solid lines represent the 1:1 line.

\section{Concluding Remarks}

From an extensive in situ data set covering about four orders of magnitude in SPM, some historical $S P M$ inversion algorithms based on different approaches have been evaluated. While these algorithms usually performed relatively well over the SPM range used for their development, they typically fail outside of these defined ranges. Because of the sensitivity of the different algorithms to the level of turbidity, generic algorithms based on a switching criteria, according to the $R_{\mathrm{rs}}$ value in the red (i.e., $670 \mathrm{~nm}$ for SeaWiFS), have been proposed in the present study. Due to its generally better performance and to its semi-analytical formulation, the SAA model has been selected as the most appropriate algorithm for the SPM assessment. Besides, compared to other approaches, SAA has the advantage to be easily adapted to the optical environment encountered. For instance, we have 
shown a great improvement in the SPM retrieval accuracy when parameters of the algorithm were adapted to the $b_{\mathrm{bp}} / S P M$ values encountered in the observed water mass. Environments with high or low $b_{\mathrm{bp}} / S P M$ values (mineral $v s$. phytoplankton dominated waters) could be distinguished from classification approaches [40,55,56], or through the use of polarization measurements [57], allowing the improvement of the SPM retrieval if such information is made available.

Taking SeaWiFS as an example, while $R_{\mathrm{rs}}(670)$ is used as the input parameter in SAA for low to medium turbid waters, $R_{\mathrm{rs}}(670)$ or $R_{\mathrm{rs}}(765)$ can be chosen for very turbid waters. The retrieval accuracy of SPM in highly turbid waters is only slightly improved when $R_{\mathrm{rs}}(765)$ is chosen instead of $R_{\mathrm{rs}}(670)$. Indeed, even if $R_{\mathrm{rs}}(765)$ presents more dynamics than $R_{\mathrm{rs}}(670)$ in very turbid waters (as explained in Section 3.1), a significant $R_{\mathrm{rs}}(670)$ vs. SPM relationship can still be established. The use of this relationship allows SPM to be estimated with a satisfactory accuracy. The preference in the SAA-HR compared to the SAA-HNIR option is thus motivated by the following reasons. First, the retrieval accuracy between the two options is very similar. Second, the signal to noise ratio in the NIR channel is generally lower than in the red [58]. This is true for SeaWiFS and MERIS but not for MODIS which presents about the same SNR in these two spectral domains. Third, while the channel centered at around $670 \mathrm{~nm}$ is available for all the sensors, this is not the case for $765 \mathrm{~nm}$ (i.e., Landsat8/OLI). At last, the retrieval accuracy of $R_{\mathrm{rs}}(765)$, obtained after the atmospheric correction procedure, has still not been estimated, as thus compared with $670 \mathrm{~nm}$. While in situ measurements have shown that SAA-HR and SAA-HNIR provide about the same SPM retrieval accuracy over very turbid waters, a first test on real satellite data over different turbid areas (not shown here)has revealed that estimated SPM values are much lower with SAA-HNIR than with SAA-HR. This pattern clearly emphasizes the necessity to better evaluate the $R_{\mathrm{rs}}$ in the near infrared spectral domain.

Acknowledgments: This study has been performed in the frame of the GlobCoastproject [59] which is funded by the Agence Nationale de la Recherche (ANR-11-BLAN-BS56-018-01). The GlobCoast project is affiliated to LOICZ and AQUIMER.When this work began, Bing HAN worked at LOG as a visiting scholar funded by the China Scholarship Council (CSC).This research is also partly funded by the National Science Fund of China (grant No.41276184), the Chinese Academy of Sciences (Nos XDA11020403, GJHZ1207), and the Key Laboratory of Marine Remote Sensing and Acoustics (grant No. KHYS1401) in Nanjing University of Information Science and Technology, China. The data in Vietnam were collected in the frame of the project VITEL funded by TOSCA (CNES, France). The authors would like to thank Bertrand Lubac to provide data of SOMLIT project. The authors acknowledge Marcel Babin for providing the COASTLOOC database, and Marcel Babin and Simon Bélanger for the data collected in June and July 2004 in the Southeastern Beaufort Sea. The authors thank K. Ruddick for the data collected in the frame of the Belcolour-2 project from cruises performed in collaboration between MUMM and LOG in 2009 and 2010.Four anonymous reviewers are acknowledged for their comments on a first version of this paper.

Author Contributions: Bing Han gathered field data and made quality check, developed final models, and finished the original manuscript and following revision. Hubert Loisel, as the major funder, proposed the idea of the work, gave permission to data in LOG and revised carefully the original manuscript. Vincent Vantrepotte gave valuable suggestions and comments, and offered valuable help in data quality control and model development. Xavier Mériaux gave much help in field data processing and in data management in LOG database. Philippe Bryère provided Gohin's model code and performed its validation. Sylvain Ouillon provided in situ data in Vietnam under the framework of VITEL, and also contributed to the revision of the manuscript. David Dessailly finished part backscattering coefficient processing and provided satellite image. Qianguo Xing provided data in China Yellow Sea. Jianhua Zhu helped in data quality check and provided part of the funding.

Conflicts of Interest: All the authors hereby declare that no conflict of interest exists in this paper.

\section{References}

1. Loisel, H.; Mangin, A.; Vantrepotte, V.; Dessailly, D.; Dinh, D.; Garnesson, P.; Ouillon, S.; Lefebvre, J.-P.; Meriaux, X.; Phan, T. Variability of suspended particulate matter concentration in coastal waters under the Mekong's influence from ocean color (MERIS) remote sensing over the last decade. Remote Sens. Environ. 2014, 150, 218-230.

2. Vanhellemont, Q.; Ruddick, K. Turbid wakes associated with offshore wind turbines observed with Landsat 8. Remote Sens. Environ. 2014, 145, 105-115. [CrossRef] 
3. Fettweis, M.; Nechad, B.; Den, E.D. An estimate of the suspended particulate matter (SPM) transport in the southern North Sea using SeaWiFS images, in situ measurements and numerical model results. Cont. Shelf Res. 2007, 27, 1568-1583. [CrossRef]

4. Doxaran, D.; Froidefond, J.M.; Lavender, S. Spectral signature of highly turbid waters: Application with SPOT data to quantify suspended particulate matter concentrations. Remote Sens. Environ. 2002, 81, 149-161. [CrossRef]

5. Toth, B.; Bodis, E. Estimation of suspended loads in the Danube River at Göd (1668 river km), Hungary. J. Hydrol. 2015, 523, 139-146. [CrossRef]

6. Pedersen, T.; Gallegos, C.; Nielsen, S. Influence of near-bottom re-suspended sediment on benthic light availability. Estuar. Coast. Shelf Sci. 2012, 106, 93-101.

7. Martin, J.M.; Windom, H.L. Present and future roles of ocean margins in regulating marine biogeochemical cycles of trace elements. In Ocean Margin Processes in Global Change; Mantoura, R.F.C., Martin, J.M., Wollast, R., Eds.; John Wiley \& Sons: Hoboken, NJ, USA, 1991; pp. 45-67.

8. Ko, F.; Baker, J. Seasonal and annual loads of hydrophobic organic contaminants from the Susquehanna River basin to the Chesapeake Bay. Mar. Pollut. Bull. 2004, 48, 840-851. [CrossRef] [PubMed]

9. Mayer, L.; Keil, R.; Macko, S. Importance of suspended participates in riverine delivery of bioavailable nitrogen to coastal zones. Glob. Biogeochem. Cycles 1998, 12, 573-579. [CrossRef]

10. Woźniak, S. Simple statistical formulas for estimating biogeochemical properties of suspended particulate matter in the southern Baltic Sea potentially useful for optical remote sensing applications. Oceanologia 2014, 56, 7-39. [CrossRef]

11. Ouillon, S.; Douillet, P.; Andrefouet, S. Coupling satellite data with in situ measurements and numerical modeling to study fine suspended-sediment transport: A study for the lagoon of New Caledonia. Coral Reefs 2004, 23, 109-122.

12. Ouillon, S.; Douillet, P.; Petrenko, A.; Neveux, J.; Dupouy, C.; Froidefond, J.M.; Andréfouët, S.; Muñoz-Caravaca, A. Optical algorithms at satellite wavelengths for total suspended matter in tropical coastal waters. Sensors 2008, 8, 4165-4185. [CrossRef]

13. Moore, G.; Aiken, J.; Lavender, S. The atmospheric correction of water colour and the quantitative retrieval of suspended particulate matter in Case II waters: Application to MERIS. Int. J. Remote Sens. 2010, 20, 1713-1733. [CrossRef]

14. Miller, R.; Mckee, B. Using MODIS Terra $250 \mathrm{~m}$ imagery to map concentrations of total suspended matter in coastal waters. Remote Sens. Environ. 2004, 93, 259-266. [CrossRef]

15. Hu, C.; Chen, Z.; Clayton, T. Assessment of estuarine water-quality indicators using MODIS medium-resolution bands: Initial results from Tampa Bay, FL, USA. Remote Sens. Environ. 2004, 93, 423-441. [CrossRef]

16. Zhang, M.; Tang, J.; Dong, Q. Retrieval of total suspended matter concentration in the Yellow and East China Seas from MODIS imagery. Remote Sens. Environ. 2010, 114, 392-403. [CrossRef]

17. Min, J.; Ryu, J.; Lee, S. Monitoring of suspended sediment variation using Landsat and MODIS in the Saemangeum coastal area of Korea. Mar. Pollut. Bull. 2012, 64, 382-390. [CrossRef] [PubMed]

18. Ondrusek, M.; Stengel, E.; Kinkade, C. The development of a new optical total suspended matter algorithm for the Chesapeake Bay. Remote Sens. Environ. 2012, 119, 243-254. [CrossRef]

19. Doxaran, D.; Froidefond, J.M.; Castaing, P. A reflectance band ratio used to estimate suspended matter concentrations in sediment-dominated coastal waters. Int. J. Remote Sens. 2010, 23, 5079-5085. [CrossRef]

20. Doxaran, D.; Froidefond, J.M.; Castaing, P. Remote-sensing reflectance of turbid sediment-dominated waters. Reduction of sediment type variations and changing illumination conditions effects by use of reflectance ratios. Appl. Opt. 2003, 42, 2623-2634. [CrossRef] [PubMed]

21. Qiu, Z. A simple optical model to estimate suspended particulate matter in Yellow River Estuary. Opt. Express 2013, 21, 27891-27904. [CrossRef] [PubMed]

22. Tassan, S. Local algorithms using SeaWiFS data for the retrieval of phytoplankton, pigments, suspended sediment, and yellow substance in coastal waters. Appl. Opt. 1994, 33, 2369-2378. [CrossRef] [PubMed]

23. Tang, J.; Wang, X.; Song, Q.; Li, T.; Chen, J.; Huang, H.; Ren, J. The statistic inversion algorithms of water constituents for the Huanghai Sea and the East China Sea. Acta Oceanol. Sin. 2004, 23, 617-626. 
24. Siswanto, E.; Tang, J.; Yamaguchi, H.; Yuhwan, A.; Ishizaka, J.; Yoo, S.; Sangwoo, K.; Kiyomoto, Y.; Yamada, K.; Chiang, C.; Kawamura, H. Empirical ocean-color algorithms to retrieve chlorophyll-a, total suspended matter, and colored dissolved organic matter absorption coefficient in the Yellow and East China Seas. J. Oceanogr. 2011, 67, 627-650. [CrossRef]

25. Dekker, A.; Vos, R.; Peters, S. Comparison of remote sensing data, model results and in situ data for total suspended matter (TSM) in the southern Frisian lakes. Sci. Total Environ. 2001, 268, 197-214. [CrossRef]

26. Nechad, B.; Ruddick, K.; Park, Y. Calibration and validation of a generic multisensor algorithm for mapping of total suspended matter in turbid waters. Remote Sens. Environ. 2010, 114, 854-866. [CrossRef]

27. Shen, F.; Zhou, Y.; Peng, X.; Chen, Y. Satellite multisensormapping of suspended particulate matter in turbid estuarine and coastal ocean, China. Int. J. Remote Sens. 2014, 35, 4173-4192. [CrossRef]

28. Kong, J.; Sun, X.; Wong, D. A semi-analytical model for remote sensing retrieval of suspended sediment concentration in the gulf of Bohai, China. Remote Sens. 2015, 7, 5373-5397. [CrossRef]

29. Gohin, F.; Loyer, S.; Lunven, M. Satellite-derived parameters for biological modelling in coastal waters: Illustration over the eastern continental shelf of the Bay of Biscay. Remote Sens. Environ. 2005, 95, $29-46$. [CrossRef]

30. Dogliotti, A.; Ruddick, K.; Nechad, B. A single algorithm to retrieve turbidity from remotely-sensed data in all coastal and estuarine waters. Remote Sens. Environ. 2015, 156, 157-168. [CrossRef]

31. Neil, C.; Cunningham, A.; Mckee, D. Relationships between suspended mineral concentrations and red-waveband reflectances in moderately turbid shelf seas. Remote Sens. Environ. 2011, 115, 3719-3730. [CrossRef]

32. Doerffer, R.; Fischer, J. Concentrations of chlorophyll suspended matter and gelbstoff in case II waters derived from satellite coastal zone color scanner data with inverse modeling methods. J. Geophys. Res. Atmos. 1994, 99, 7457-7466. [CrossRef]

33. Volpe, V.; Silvestri, S.; Marani, M. Remote sensing retrieval of suspended sediment concentration in shallow waters. Remote Sens. Environ. 2011, 115, 44-54. [CrossRef]

34. Bowers, D.G.; Binding, C.E. The optical properties of mineral suspended particles: A review and synthesis. Estuar. Coast. Shelf Sci. 2006, 67, 219-230. [CrossRef]

35. Snyder, W.; Arnone, R.; Davis, C. Optical scattering and backscattering by organic and inorganic particulates in U.S. coastal waters. Appl. Opt. 2008, 47, 666-677. [CrossRef] [PubMed]

36. Woźniak, S.; Stramski, D.; Stramska, M. Optical variability of seawater in relation to particle concentration, composition, and size distribution in the nearshore marine environment at Imperial Beach, California. J. Geophys. Res. 2010, 115. [CrossRef]

37. Neukermans, G.; Loisel, H.; Meriaux, X. In situ variability of mass-specific beam attenuation and backscattering of marine particles with respect to particle size, density, and composition. Limnol. Oceanogr. 2012, 57, 124-144. [CrossRef]

38. Babin, M.; Morel, A.; Fourniersicre, V. Light scattering properties of marine particles in coastal and open ocean waters as related to the particle mass concentration. Limnol. Oceanogr. 2003, 48, 843-859. [CrossRef]

39. Doron, M.; Babin, M.; Mangin, A.; Fantond'Andon, O. Estimation of light penetration, and horizontal and vertical visibility in oceanic and coastal waters from surface reflectance. J. Geophys. Res. 2006, 112, C06003. [CrossRef]

40. Lubac, B.; Loisel, H. Variability and classification of remote sensing reflectance spectra in the eastern English Channel and southern North Sea. Remote Sens. Environ. 2007, 110, 45-58. [CrossRef]

41. Bélanger, S.; Babin, M.; Larouche, P. An empirical ocean color algorithm for estimating the contribution of chromophoric dissolved organic matter to total light absorption in optically complex waters. J. Geophys. Res. 2008, 113, C04027. [CrossRef]

42. Mueller, J.L.; Davis, D.; Arnone, R.; Frouin, R.; Carder, K.L.; Lee, Z.P.; Steward, R.G.; Hooker, S.; Mobley, C.D.; McLean, S. Above-water radiance and remote sensing reflectance measurement and analysis protocols. In Ocean Optics Protocols for Satellite Ocean Color Sensor Validation; Mueller, J.L., Fargion, G.S., Eds.; NASAGoddard Space Flight Center: Washington, DC, USA, 2002; pp. 171-182.

43. Mueller, J.L. In-water radiometric profile measurements and data analysis protocols. In Ocean Optics Protocols for Satellite Ocean Color Sensor Validation; Mueller, J.L., Fargion, G.S., McClain, C.R., Eds.; NASA Goddard Space Flight Center: Greenbelt, MD, USA, 2003; Volume 3, pp. 7-20. 
44. Loisel, H.; Morel, A. Non-isotropy of the upward radiance field in typical coastal (Case 2) waters. Int. J. Remote Sens. 2010, 22, 275-295. [CrossRef]

45. Loisel, H.; Lubac, B.; Dessailly, D. Effect of inherent optical properties variability on the chlorophyll retrieval from ocean color remote sensing: An in situ approach. Opt. Express 2010, 18, 20949-20959. [CrossRef] [PubMed]

46. Doxaran, D.; Cherukuru, R.; Lavender, S. Use of Reflectance band ratios to estimate suspended and dissolved matter concentrations in Estuarine Waters. Int. J. Remote Sens. 2005, 26, 1763-1769. [CrossRef]

47. Doxaran, D.; Devred, E.; Babin, M. A 50\% increase in the mass of terrestrial particles delivered by the mackenzie river into the Beaufort Sea (Canadian Arctic Ocean) over the last 10 years. Biogeosciences 2015, 12, 3551-3565. [CrossRef]

48. Gohin, F. Annual cycles of chlorophyll-a, non-algal suspended particulate matter, and turbidity observed from space and in-situ in coastal waters. Ocean Sci. 2011, 7, 705-732. [CrossRef]

49. Gohin, F.; Druon, J.; Lampert, L. A five channel chlorophyll concentration algorithm applied to SeaWiFS data processed by SeaDAS in coastal waters. Int. J. Remote Sens. 2010, 23, 1639-1661. [CrossRef]

50. Campbell, J. The lognormal distribution as a model for bio-optical variability in the sea. J. Geophys. Res. 1995, 100, 13237-13254. [CrossRef]

51. Binding, C.; Bowers, D.; Mitchelsonjacob, E. Estimating suspended sediment concentrations from ocean colour measurements in moderately turbid waters; the impact of variable particle scattering properties. Remote Sens. Environ. 2005, 94, 373-383. [CrossRef]

52. Goyens, C.; Jamet, C.; Schroeder, T. Evaluation of four atmospheric correction algorithms for MODIS-Aqua images over contrasted coastal waters. Remote Sens. Environ. 2013, 131, 63-75. [CrossRef]

53. Spectral Response Functions and Bandpass Averaged Quantities. Available online: http:/ / oceancolor.gsfc.nasa.gov/DOCS/RSR_tables.html (accessed on 3 March 2016).

54. Ouillon, S.; Forget, P.; Froidefond, J.M.; Naudin, J.J. Estimating suspended matter concentrations from SPOT data and from field measurements in the Rhône river plume. Mar. Tech. Soc. J. 1997, 31, 15-20.

55. Vantrepotte, V.; Brunet, C.; Meriaux, X.; Lecuyer, E.; Vellucci, V.; Santer, R. Bio-optical properties of coastal waters in the Eastern English Channel. Estuar. Coast. Shelf Sci. 2007, 72, 201-212. [CrossRef]

56. Lahet, F.; Ouillon, S.; Forget, P. Colour classification of coastal waters of Ebro river plume from spectral reflectances. Int. J. Remote Sens. 2001, 22, 1639-1664. [CrossRef]

57. Loisel, H.; Duforet, L.; Dessailly, D. A first attempt to assess marine particles composition from remote sensing: Exploitation of the POLDER polarized radiances. Opt. Soc. Am. 2006. [CrossRef]

58. Jolivet, D.; Ramon, D.; Deschamps, P.Y.; Steinmetz, F.; Fougnie, B.; Henry, P. How the ocean color products is limited by atmospheric correction. In Proceedings of the Envisat Symposium 2007, Montreux, Switzerland, 23-27 April 2007.

59. Gloabcoast. Available online: http:/ /www.foresea.fr/globcoast (accessed on 3 March 2016).

(C) 2016 by the authors; licensee MDPI, Basel, Switzerland. This article is an open access article distributed under the terms and conditions of the Creative Commons by Attribution (CC-BY) license (http://creativecommons.org/licenses/by/4.0/). 